\title{
Spinal Fbxo3-Dependent Fbxl2 Ubiquitination of Active Zone Protein RIM1 $\alpha$ Mediates Neuropathic Allodynia through $\mathrm{Ca}_{\mathrm{V}} 2.2$ Activation
}

\author{
(C) Cheng-Yuan Lai, ${ }^{1,2 *}$ Yu-Cheng Ho, ${ }^{2 *}$ Ming-Chun Hsieh, ${ }^{2,3}$ Hsueh-Hsiao Wang, ${ }^{2}$-Jen-Kun Cheng, ${ }^{2,4}$ Yat-Pang Chau, ${ }^{2}$ \\ and ${ }^{\circ}$ Hsien-Yu Peng ${ }^{2}$ \\ ${ }^{1}$ Department of Veterinary Medicine, College of Veterinary Medicine, National Chung-Hsing University, Taichung 40227, Taiwan, ${ }^{2}$ Department of \\ Medicine, Mackay Medical College, New Taipei 25160, Taiwan, ${ }^{3}$ Department of Physiology, College of Medicine, National Taiwan University, Taipei 10051, \\ Taiwan, and ${ }^{4}$ Department of Anesthesiology, Mackay Memorial Hospital, Taipei 10449, Taiwan
}

\begin{abstract}
Spinal plasticity, a key process mediating neuropathic pain development, requires ubiquitination-dependent protein turnover. Presynaptic active zone proteins have a crucial role in regulating vesicle exocytosis, which is essential for synaptic plasticity. Nevertheless, the mechanism for ubiquitination-regulated turnover of presynaptic active zone proteins in the progression of spinal plasticity-associated neuropathic pain remains unclear. Here, after research involving Sprague Dawley rats, we reported that spinal nerve ligation (SNL), in addition to causing allodynia, enhances the Rab3-interactive molecule-1 $\alpha$ (RIM1 $\alpha$ ), a major active zone protein presumed to regulate neural plasticity, specifically in the synaptic plasma membranes (SPMs) of the ipsilateral dorsal horn. Spinal RIM1 $\alpha$-associated allodynia was mediated by Fbxo3, which abates Fbxl2-dependent RIM1 $\alpha$ ubiquitination. Subsequently, following deubiquitination, enhanced RIM1 $\alpha$ directly binds to CaV2.2, resulting in increased CaV2.2 expression in the SPMs of the dorsal horn. While exhibiting no effect on Fbxo3/Fbxl2 signaling, the focal knockdown of spinal RIM1 $\alpha$ expression reversed the SNL-induced allodynia and increased spontaneous EPSC (sEPSC) frequency by suppressing RIM1 $\alpha$-facilitated $\mathrm{Ca}_{\mathrm{V}} 2.2$ expression in the dorsal horn. Intrathecal applications of BC-1215 (a Fbxo3 activity inhibitor), Fbxl2 mRNA-targeting small-interfering RNA, and $\omega$-conotoxin GVIA (a Ca 2.2 blocker) attenuated RIM1 $\alpha$ upregulation, enhanced RIM1 $\alpha$ expression, and exhibited no effect on RIM1 $\alpha$ expression, respectively. These results confirm the prediction that spinal presynaptic Fbxo3-dependent Fbxl2 ubiquitination promotes the subsequent RIM $1 \alpha / \mathrm{Ca}_{\mathrm{V}} 2.2$ cascade in SNL-induced neuropathic pain. Our findings identify a role of the presynaptic active zone protein in pain-associated plasticity. That is, RIM1 $\alpha$ facilitated $\mathrm{Ca}_{\mathrm{v}} 2.2$ expression plays a role in the downstream signaling of Fbxo3-dependent Fbxl2 ubiquitination/degradation to promote spinal plasticity underlying the progression of nociceptive hypersensitivity following neuropathic injury.
\end{abstract}

Key words: Cav2.2; Fbxl2; Fbxo3; neuropathic pain; Rim1 $\alpha$

Significance Statement

Ubiquitination is a well known process required for protein degradation. Studies investigating pain pathology have demonstrated that ubiquitination contributes to chronic pain by regulating the turnover of synaptic proteins. Here, we found that the spinal presynaptic active zone protein Rab3-interactive molecule- $1 \alpha(\mathrm{RIM} 1 \alpha)$ participates in neuropathic pain development by binding to and upregulating the expression of $\mathrm{Ca}_{\mathrm{v}} 2.2$. In addition, Fbxo3 modifies this pathway by inhibiting Fbxl2-mediated RIM1 $\alpha$ ubiquitination, suggesting that presynaptic protein ubiquitination makes a crucial contribution to the development of neuropathic pain. Research in this area, now in its infancy, could potentially provide a novel therapeutic strategy for pain relief.

\section{Introduction}

The presynaptic active zone mediates synaptic vesicle exocytosis. Modulation of the zone's molecular composition is important for the regulation of neurotransmitter release, synaptic plasticity, and neuronal network activity (Spangler et al., 2013; Michel et al., 2015). Rab3-interactive molecule- $1 \alpha(\operatorname{RIM} 1 \alpha)$ is a major RIM isoform and has been identified as an active zone protein with the ability to significantly regulate vesicle exocytosis (Geppert et al., 
1997; Wang et al., 1997, 2000). Electrophysiological recordings of pyramidal neurons dissected from $R I M 1 \alpha$-knock-out (KO) mice reveal that RIM $1 \alpha$ is essential for synaptic vesicle priming and presynaptic long-term plasticity (Kaeser et al., 2008). In the Schaffer collateral-CA1 synapse, RIM $1 \alpha$ is recognized as a central molecule integrating active zone proteins and maintaining the probability of vesicle release during short-term synaptic plasticity (Schoch et al., 2002). Although ample studies have linked presynaptic proteins to plasticity underlying the nociceptive hypersensitivity after neuropathic injury (Kohno et al., 2005; Yan et al., 2013), the potential role of the spinal RIM $1 \alpha$-associated presynaptic plasticity in the development of neuropathic pain remains unestablished to date.

Vesicle exocytosis at the active zone is initiated by the influx of $\mathrm{Ca}^{2+}$ through $\mathrm{Ca}^{2+}$ channels (Gandini et al., 2011). In the CA1 area of the hippocampus, evidence obtained from field potentials and whole-cell voltage-clamp recordings has confirmed the essential role of RIM $1 \alpha$ in $\mathrm{Ca}^{2+}$-triggered exocytosis (Schoch et al., 2006). Notably, RIM has been demonstrated to associate with $\mathrm{N}$-type voltage-dependent calcium channels $\left(\mathrm{Ca}_{\mathrm{V}} 2.2\right)$, thereby enabling fast and synchronous neurotransmitter release at the presynaptic site (Coppola et al., 2001; Hibino et al., 2002). In addition, spinal $\mathrm{Ca}_{\mathrm{V}} 2.2$ has been linked to the development of neuropathic pain because mice lacking $\mathrm{Ca}_{\mathrm{V}} 2.2$ have decreased tactile allodynia caused by nerve ligation (Saegusa and Tanabe, 2014). Conversely, the spinal administration of $\omega$-conotoxin, a selective $\mathrm{Ca}_{\mathrm{V}} 2.2$ blocker, produces a dose-dependent amelioration of the nerve injury-induced mechanical allodynia (Jayamanne et al., 2013). These experiments have given rise to the plausible hypothesis that spinal $\operatorname{RIM} 1 \alpha$ may recruit nearby $\mathrm{Ca}_{\mathrm{V}} 2.2$ and thereby regulate neurotransmitter release at the presynaptic terminal to participate in the neural plasticity underlying the development of neuropathic pain.

Ubiquitination-associated protein degradation has attracted researchers' attention as a mechanism regulating the abundance of synaptic proteins (Varshavsky, 2005; Yi and Ehlers, 2005). Ubiquitination-dependent protein turnover has been linked to changes in the composition of synaptic proteins in response to altered synaptic activity (Ehlers, 2003; Yi and Ehlers, 2005). Interestingly, previous studies have demonstrated that Fbxl2, a synapse-localized E3 ubiquitin ligase implicated in neural transmission, directly binds to RIM1 and subsequently results in RIM1 ubiquitination (Yao et al., 2007, 2011). In Fbxl2-KO mice, RIM1 has a longer half-life, and it has the ability to significantly reduce ubiquitination in association with an enhanced frequency of miniature EPSCs (mEPSCs) in the CA3-CA1 synapse (Yao et al., 2007). Recently, studies have revealed that Fbxo3, another E3ubiquitin ligase subunit, potently stimulates cytokine secretion by destabilizing Fbxl2 (Chen et al., 2013; Mallampalli et al., 2013). Together, these studies link the Fbxo3/Fbxl2 cascade to RIM1 $\alpha$ unubiquitination and activation. As RIM $1 \alpha$ is known to be essential for $\mathrm{Ca}_{\mathrm{V}}$ 2.2-associated vesicle exocytosis (Schoch et al., 2006) and our laboratory has demonstrated that Fbxo3/Fbxl2 signaling contributes to the spinal plasticity underlying neuropathic pain (Lin et al., 2015b), we wondered whether the Fbxo3/ Fbl2 cascade contributes to the spinal plasticity underlying

${ }^{*}$ C.-Y.L. and Y.-C.H contributed equally to this work. The authors declare no competing financial interests.

Correspondence should be addressed to Hsien-Yu Peng, Department of Medicine, Mackay Medical College, Number 46, Section 3, Zhongzheng Road, Sanzhi District, New Taipei 25245, Taiwan. E-mail: hsien.yu@gmail.com. DOI:10.1523/JNEUROSCI.1732-16.2016

Copyright $\odot 2016$ the authors $\quad 0270-6474 / 16 / 369723-17 \$ 15.00 / 0$ neuropathic pain development by affecting RIM $1 \alpha$ and the subsequent $\mathrm{Ca}_{\mathrm{V}}$ 2.2-dependent neurotransmission.

Here, we identify the role of spinal RIM $1 \alpha$ in neuropathic pain processing in the pre-SPMs of the dorsal horn. We conclude that Fbxo3 contributes to the spinal plasticity underlying the development of neuropathic pain by directly binding to and ubiquitinating the Fbxl2, which results in the uncoupling of RIM1 $\alpha$ from Fbxl2 and subsequently activates RIM1 $\alpha$ to recruit $\mathrm{Ca}_{\mathrm{V}} 2.2$ to the presynaptic site of the dorsal horn.

\section{Materials and Methods}

Animal preparations. Adult male Sprague Dawley rats weighing 200-250 g were used. They were housed at room temperature $\left(23 \pm 2^{\circ} \mathrm{C}\right)$ with a $12 \mathrm{~h}$ light/dark cycle (lights on from 8:00 A.M. to 8:00 P.M.), and were fed food and water ad libitum. All animal procedures in this study were conducted in accordance with the guidelines of the International Association for the Study of Pain (Zimmermann, 1983) and were reviewed and approved by the Institutional Review Board of Taipei Medical University, Taipei, Taiwan.

Spinal nerve ligation. Ligations of spinal nerves in rats were performed under isoflurane anesthesia (induction 5\%, maintenance $2 \%$ in oxygen), following the methods of a previous study (Lin et al., 2016). After isoflurane anesthesia, rats were placed in a prone position. An incision was made, and the left L5-L6 spinal nerves were carefully isolated and tightly ligated with 6-0 silk sutures $2-5 \mathrm{~mm}$ distal to the dorsal root ganglia. Finally, the wound and the surrounding skin were sutured. In the sham-operation group, the surgical procedures were identical to those in the nerve-ligation group, except the silk sutures were left unligated.

Intrathecal catheter. Implantation of intrathecal cannula was performed as described in our previous studies (Lin et al., 2015a,b). Under isoflurane anesthetized (induction 5\%, maintenance $2 \%$ in oxygen), PE-10 Silastic tubing was implanted in the lumbar enlargement of the spinal cord. The outer part of the catheter was plugged and immobilized onto the skin on closure of the wound. After implantation, the animals were allowed to recover for $3 \mathrm{~d}$. Rats showed neurological deficits after surgery were killed and excluded from statistical analyses.

Behavioral studies. Tactile sensitivity was assessed by measuring each rat's paw-withdrawal threshold in response to probing with von Frey monofilaments (Stoelting) according to the method of Schäfers (Schäfers et al., 2003). In brief, rats were placed individually in an opaque plastic cylinder, which was placed on a wire mesh. Animals were habituated for $1 \mathrm{~h}$ to allow acclimatization to the test environment before each test. After acclimatization, calibrated von Frey filaments $(0.07-26.0 \mathrm{~g})$ were applied to the plantar surfaces of the hindpaws of rats. The withdrawal threshold was assayed $1 \mathrm{~d}$ before surgery to ensure that the animals had normal tactile sensitivity. Because a previous study has showed the mean withdrawal threshold of naive rats are approximately $15 \mathrm{~g}$ (Chung et al., 2004), rats that displayed thresholds lower than $15 \mathrm{~g}$ (which could be sensitive to nonpainful stimulation) and exhibited no withdrawal when the hair of $15 \mathrm{~g}$ was applied (which could be insensitive to painful stimulation) were excluded for further experiments. Motor function was assessed using an accelerating rotarod apparatus (LE8500, Ugo Basile). For acclimatization, the animals were subjected to three training trials at 3-4 $\mathrm{h}$ intervals on 2 separate days. During the training sessions, the rod was set to accelerate from 3 to $30 \mathrm{rpm}$ over a $180 \mathrm{~s}$ period. During the test session, the performance times of rats were recorded up to a cutoff time of $180 \mathrm{~s}$. Three measurements were obtained at intervals of $5 \mathrm{~min}$ and were averaged for each test.

Subcellular fractionation. The dissected dorsal horn (L4-L5) sample was homogenized in $25 \mathrm{~mm}$ Tris- $\mathrm{HCl}, 150 \mathrm{~mm} \mathrm{NaCl}, 1 \% \mathrm{NP}-40,1 \%$ sodium deoxycholate, and $0.1 \%$ SDS with a complete protease inhibitor mixture (Roche). After incubation on ice $(1 \mathrm{~h})$, the lysates were centrifuged $\left(14,000 \mathrm{rpm}, 20 \mathrm{~min}, 4^{\circ} \mathrm{C}\right)$. The supernatant is recognized as total homogenate. Subcellular fractions were prepared and modified according to the method described in Butz et al. (1999). Briefly, rats were anesthetized and decapitated. The spinal cords were dissected on ice. All the subcellular fractionation procedures and subsequent handling of frac- 
A

IB:RIM1 $\alpha$

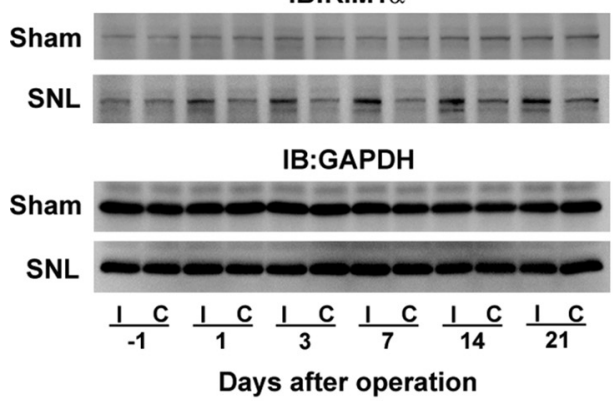

B

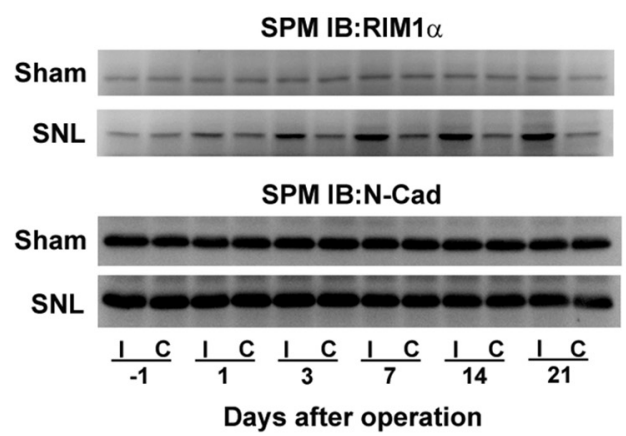

C

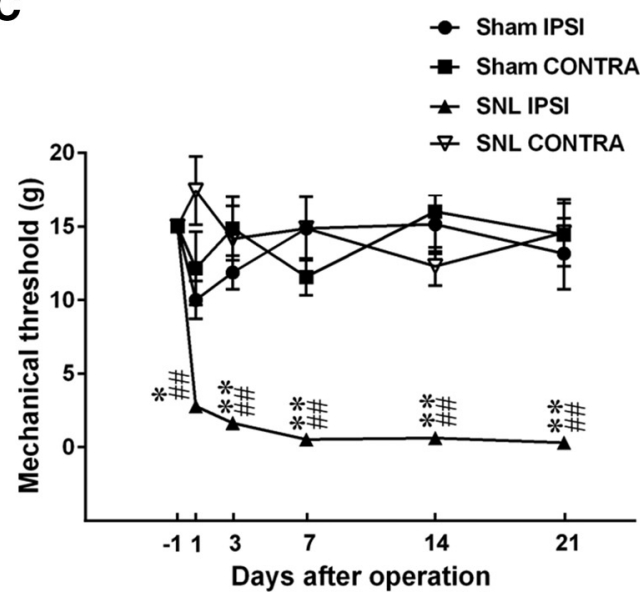

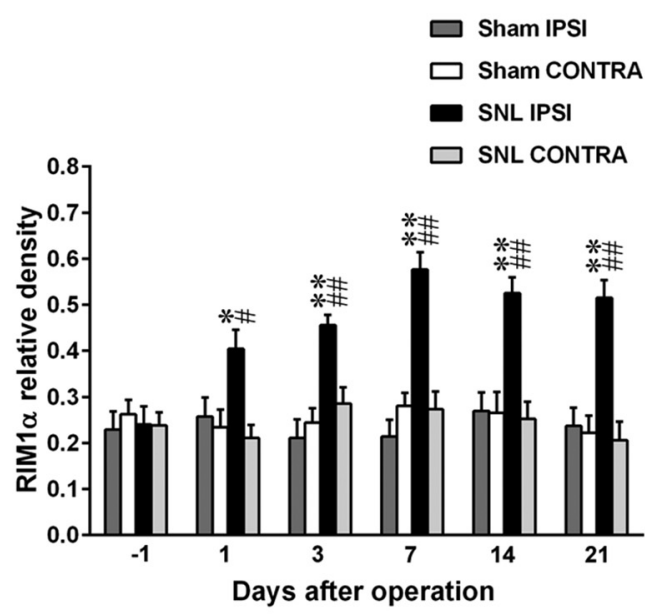

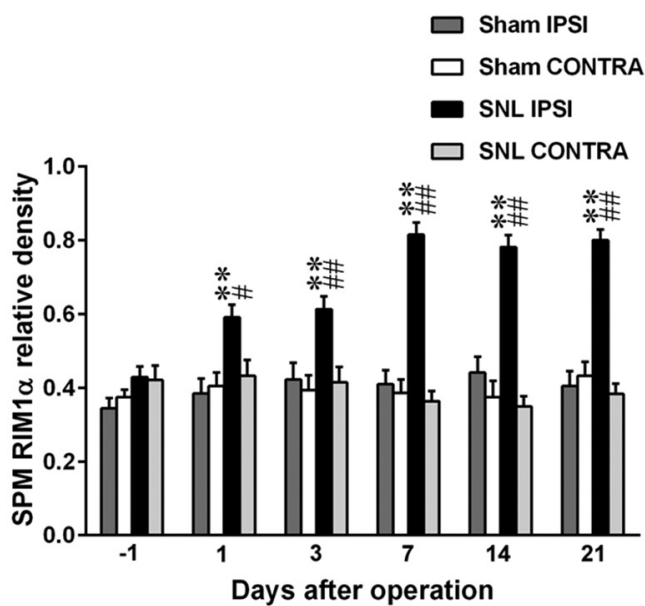

Figure 1. SNL upregulates RIM1 $\alpha$ expression in SPMs of the dorsal horn accompanied with allodynia. $\boldsymbol{A}, \boldsymbol{B}$, Representative Western blotting and statistical analysis (normalized to GAPDH and N-cadherin) revealing SNL-increased RIM1 $\alpha$ expression in the total homogenate $(\boldsymbol{A})$ and SPMs $(\boldsymbol{B})$ of the ipsilateral (I and IPSI), but not the contralateral (C and CONTRA), dorsal horn. IB, Homogenate immunoblotting; SPM IB, SPM immunoblotting. Homogenate, two-way ANOVA with repeated measures over time, treatment, $F_{(3,20)}=33.35, p<0.001 ;$ time, $F_{(5,100)}=3.806, p=$ 0.003; treatment $\times$ time, $F_{(15,100)}=3.164, p<0.001$; SPMs, two-way ANOVA with repeated measures over time, treatment, $F_{(3,20)}=87.35, p<0.001 ;$ time, $F_{(5,100)}=5.201, p<0.001$; treatment $\times$ time, $F_{(15,100)}=5.091, p<0.001 ;{ }^{*} p<0.05,{ }^{* *} p<0.01$ vs Sham IPSI. \#p $<0.05$, \#\#p $<0.01$ vs SNL Day $-1 ; n=6$. C, Time course of SNL-reduced withdrawal threshold of the ipsilateral hindpaw (von Frey test). Two-way ANOVA with repeated measures over time, treatment, $F_{(3,24)}=59.25, p<0.001 ;$ time, $F_{(5,120)}=4.81, p<0.001$; treatment $\times$ time, $F_{(15,120)}=3.79$, $p<0.001 ;{ }^{*} p<0.05,{ }^{* *} p<0.01$ vs Sham IPSI; ${ }^{\# \#} p<0.01$ vs Day $-1 . n=7$.

tionated samples were done at $4^{\circ} \mathrm{C}$. The spinal cords were homogenized in ice-cold sucrose/HEPES buffer ( $0.32 \mathrm{~m}$ sucrose, $10 \mathrm{~mm}$ HEPES, $\mathrm{pH}$ 7.4) containing protease inhibitors and phosphatase inhibitor, and centrifuged at $800 \times g$ for $10 \mathrm{~min}$. Supernatant (S1) was separated from pellet containing nuclei plus debris (P1). Collected supernatant S1 was centrifuged at $9000 \times g$ for 20 min to obtain supernatant S2 and crude synaptosomal fraction from pellet $\mathrm{P} 2$. The pellet $\mathrm{P} 2$ was washed once in sucrose/HEPES buffer, lysed hypotonically, and centrifuged at 25,000 $\times$ $g$ for 20 min to isolate synaptosomal membranes in the pellet (LP1), whereas the free synaptic vesicles remained in the supernatant (LS1). The 
A
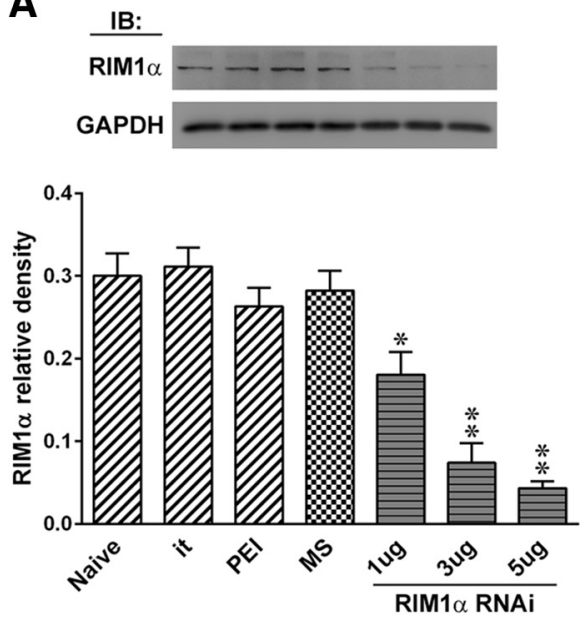

C
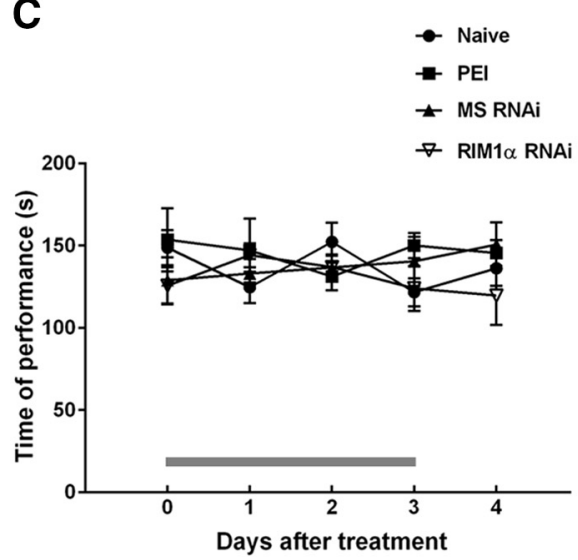

$\mathbf{F}$
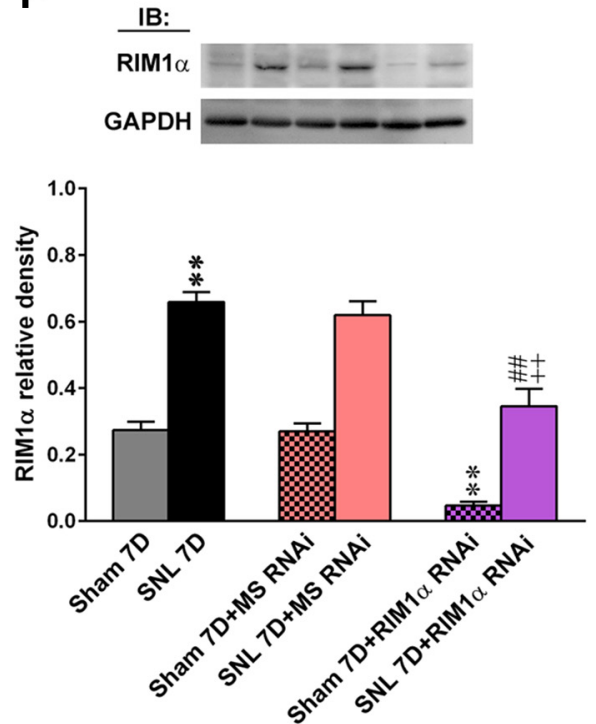

B
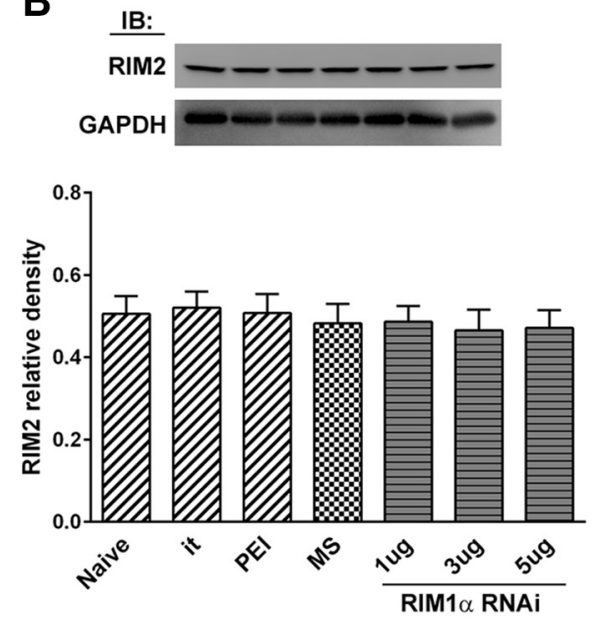

D

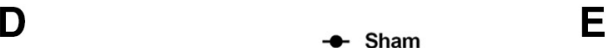

- Sham

- Sham+it

E

^ Sham+it+MS RNA

- SNL+it

^ SNL+it+MS RNAi

$\rightarrow$ Sham+it+RIM1 $\alpha$ RNA
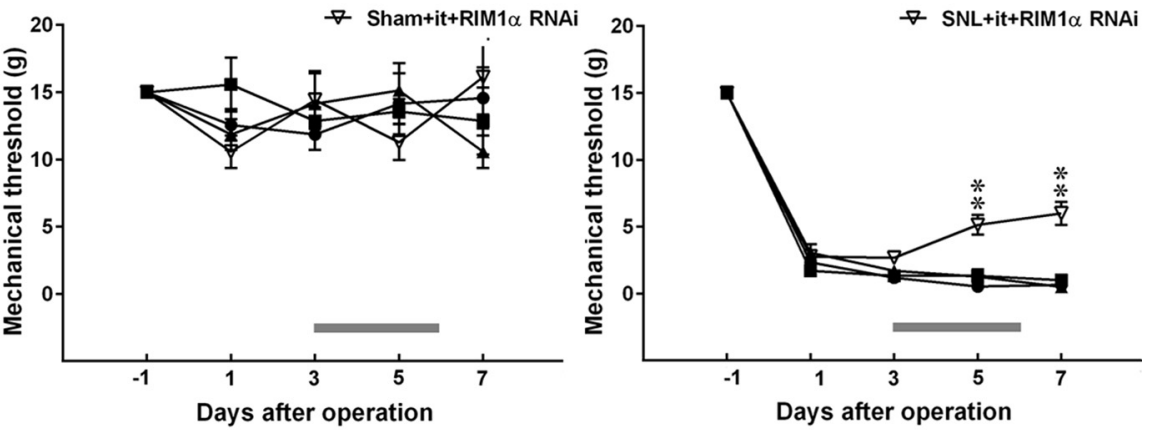

G

SPM IB:

$\operatorname{RIM1} \alpha-\ldots-\cdots$

$\mathrm{N}$-Cad

$-\infty-\infty$

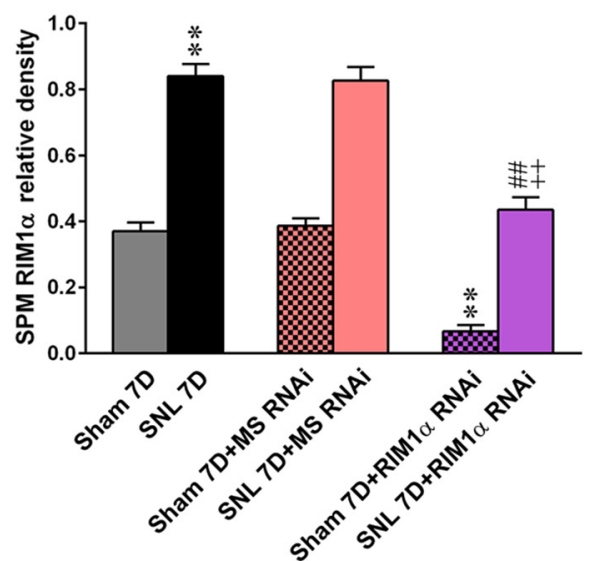

Figure 2. The knockdown of spinal RIM1 $\alpha$ expression relieves SNL-induced allodynia. $\boldsymbol{A}, \boldsymbol{B}$, Representative Western blot and statistical analysis (normalized to GAPDH) demonstrating that the intrathecal administration of RIM1 $\alpha$ mRNA-targeting siRNA (RIM1 $\alpha$ RNAi; 1, 3, and $5 \mu \mathrm{g} ; 10 \mu \mathrm{l}$; once daily for $4 \mathrm{~d}$ ), but not missense siRNA (MS; $5 \mu \mathrm{g}, 10 \mu \mathrm{l}$ ) or polyethylenimine (a transfection reagent, PEl; $10 \mu \mathrm{l})$, dose-dependently decreased spinal RIM1 $\alpha$ expression of naive rat. Yet, all these treatments failed to affect the abundance of RIM2 in the dorsal horn sample of naive rat. it, Implantation of an intrathecal catheter; IB, immunoblotting. RIM1 $\alpha$, one-way ANOVA, post hoc Tukey's test, $F_{(6,35)}=22.96, p<0.001$; RIM2, one-way ANOVA, post hoc Tukey's test, $F_{(6,35)}=$ $0.2130, p=0.970 ;{ }^{*} p<0.05,{ }^{* *} p<0.01$ vs Naive. $n=6$. C, The intrathecal application of neither RIM1 $\alpha$ mRNA-targeting siRNA (RIM1 $\alpha$ RNAi; $5 \mu \mathrm{g}, 10 \mu$ ) nor missense siRNA (MS RNAi; $5 \mu \mathrm{g}$, $10 \mu \mathrm{l}$ ) resulted in motor deficits in rats (rotarod test). The gray bar at the bottom indicates the duration of intrathecal administration. Two-way ANOVA with repeated measures over time, treatment, $F_{(3,24)}=2.27, p=0.11 ;$ time, $F_{(4,96)}=0.11, p=0.98$; treatment $\times$ time, $F_{(12,96)}=0.86, p=0.59 ; n=7 . \mathbf{D}, \boldsymbol{E}$, Although it exhibited no effect on sham-operated animals, intrathecal RIM1 $\alpha$ mRNA-targeting siRNA (SNL 7D + it + RIM1 $\alpha$ RNAi; $5 \mu \mathrm{g}, 10 \mu \mathrm{l}$ ) increased the withdrawal threshold of SNL animals on postoperative days 5 and 7 (von Frey test). The gray bar at the bottom indicates the duration of intrathecal administration. In sham-operated animals, two-way ANOVA with repeated measures over time, treatment, $F_{(3,24)}=0.10$, (Figure legend continues.) 
pellet LP1 was resuspended with $1.1 \mathrm{~m}$ sucrose/HEPES buffer, layered on the bottom of a discontinuous sucrose gradient $(0.855$ and $0.32 \mathrm{M})$ and centrifuged for $2.5 \mathrm{~h}$ at 19,000 rpm, resulting in the isolation of myelin (in the $0.32 / 0.855 \mathrm{M}$ sucrose interface), SPMs (in the $0.855 / 1.1 \mathrm{M}$ sucrose interface), and mitochondria (in the pellet). All protein concentrations were determined with the BCA protein assay kit (Pierce), using BSA as a standard.

Western blotting. The sample was separated on an acrylamide gel and transferred to a polyvinylidene difluoride membrane, which was then incubated ( $1 \mathrm{~h}$, room temperature) in either rabbit anti-RIM1 $\alpha$ (1:250; Biorbyt), rabbit anti-RIM2 (1:400; GeneTex), goat anti-Fbxl2 antibody (1:250; Santa Cruz Biotechnology), rabbit anti-Fbxo3 antibody (1:500; Santa Cruz Biotechnology), mouse anti-ubiquitin antibody (1:1000; Santa Cruz Biotechnology), rabbit anti-Ca 2.2 (1:200; Thermo Fisher Scientific), N-cadherin (mouse; 1:2000; Thermo Fisher Scientific), or mouse anti-glyceraldehyde 3-phosphate dehydrogenase (GAPDH; 1:4000; Santa Cruz Biotechnology). The blots were washed and incubated ( $1 \mathrm{~h}$, room temperature) in peroxidase-conjugated goat antirabbit IgG (1:8000; Jackson ImmunoResearch) or goat anti-mouse IgG (1:8000; Jackson ImmunoResearch). The protein bands were visualized using an enhanced chemiluminescence detection kit (ECL Plus, Millipore) and then subjected to a densitometric analysis with Science Lab 2003 (Fuji).

Coprecipitation studies. Extractions of dorsal horn samples were incubated with a rabbit anti-RIM1 $\alpha$ (Biorbyt), goat anti-Fbxl2 antibody (Santa Cruz Biotechnology), or rabbit anti-Ca 2.2 (Thermo Fisher Scientific) overnight at $4^{\circ} \mathrm{C}$. The 1:1 protein agarose suspension (Millipore) slurry was added to the protein immunocomplex, and the mixture was incubated at $4^{\circ} \mathrm{C}$ for $2-3 \mathrm{~h}$. Agarose beads were washed once with $1 \%$ $(\mathrm{v} / \mathrm{v})$ Triton X-100 in immunoprecipitation buffer [50 mM Tris-Cl, $\mathrm{pH}$ 7.4, $5 \mathrm{~mm}$ EDTA, $0.02 \%(\mathrm{w} / \mathrm{v})$ sodium azide], twice with $1 \%(\mathrm{v} / \mathrm{v})$ Triton $\mathrm{X}-100$ in immunoprecipitation buffer plus $300 \mathrm{~mm} \mathrm{NaCl}$, and three times with immunoprecipitation buffer only. Bound proteins were eluted with SDS polyacrylamide gel electrophoresis sample buffer at $95^{\circ} \mathrm{C}$. The proteins were then separated using SDS-PAGE, electrophoretically transferred onto polyvinylidene difluoride membranes, and detected using a rabbit anti-RIM1 $\alpha$ (1:250; Biorbyt), mouse anti-ubiquitin antibody (1: 1000; Santa Cruz Biotechnology), or rabbit anti-Ca 2.2 (1:200; Thermo Fisher Scientific).

Spinal slice preparations. Under anesthesia with isoflurane, rats underwent laminectomy for removal of the lumbar spinal cord. The lumbar spinal cord section was placed in ice-cold sucrose artificial CSF (aCSF) bubbled with $95 \% \mathrm{O}_{2} / 5 \% \mathrm{CO}_{2}$. The sucrose aCSF consisted of the following (in $\mathrm{mm}$ ): 234 sucrose, $3.6 \mathrm{KCl}, 1.2 \mathrm{MgCl}_{2}, 2.5 \mathrm{CaCl}_{2}, 1.2$ $\mathrm{NaH}_{2} \mathrm{PO}_{4}, 12$ glucose, and $25 \mathrm{NaHCO}_{3}$. The pia-arachnoid membrane was removed from the section. The spinal cord tissue was then placed in a shallow groove formed in a gelatin block and glued on the stage of a vibratome (DTK1000, Dosaka). Transverse spinal cord slices $(400 \mu \mathrm{m})$ were cut at the L5-L6 level in the ice-cold sucrose aCSF. After dissection, slices were equilibrated in aCSF at room temperature for $\geq 1 \mathrm{~h}$ before recording. The aCSF consisted of the following (in $\mathrm{mm}$ ): $117 \mathrm{NaCl}, 4.5$ $\mathrm{KCl}, 2.5 \mathrm{CaCl}_{2}, 1.2 \mathrm{MgCl}_{2}, 1.2 \mathrm{NaH}_{2} \mathrm{PO}_{4}, 25 \mathrm{NaHCO}_{3}$, and 11.4 dextrose bubbled with $95 \% \mathrm{O}_{2} / 5 \% \mathrm{CO}_{2}, \mathrm{pH} 7.4$. During recordings, one slice was mounted on a submerged recording chamber and continuously perfused with oxygenated aCSF at 3-4 $\mathrm{ml} / \mathrm{min}$.

\footnotetext{
(Figure legend continued.) $\quad p=0.96$; time, $F_{(4,96)}=1.23, p=0.30$; treatment $\times$ time, $F_{(12,96)}=1.45, p=0.16$; in SNL animals, two-way ANOVA with repeated measures over time, treatment, $F_{(3,24)}=20.83, p<0.001$; time, $F_{(4.96)}=961.9, p<0.001$; treatment $\times$ time, $F_{(12,96)}=10.62, p<0.001 ;{ }^{* *} p<0.01$ vs SNL; $n=7 . \boldsymbol{F}, \mathbf{G}$, Representative Western blotting and statistical analysis demonstrating that the RIM1 $\alpha$ expression in the total homogenate $(\boldsymbol{F}$, normalized to GAPDH) and synaptic plasma membranes ( $\boldsymbol{G}$, normalized to $\mathrm{N}$-cadherin) of the ipsilateral dorsal horn on postoperative day 7 of sham-operated and SNL animals were attenuated by the administration of RIM1 $\alpha$ mRNA-targeting siRNA (SNL 7D + RIM1 $\alpha$ RNAi, $5 \mu \mathrm{g}, 10 \mu \mathrm{l}$; once daily at days $3-6$ after SNL). IB, Homogenate immunoblotting; SPM IB, SPM immunoblotting. Student's $t$ tests; ${ }^{* *} p<0.01$ vs Sham 7D; \#\#p $<0.01$ vs SNL 7D. $++p<$ 0.01 vs Sham $7 \mathrm{D}+\mathrm{RIM} 1 \alpha$ RNAi; $n=6$.
}

Whole-cell electrophysiological recordings. The spinal lamina II was identified by a translucent band in the superficial dorsal horn on an upright fixed-stage infrared-differential interference contrast microscope (BX51WI, Olympus). Spinal lamina I and outer lamina II were selected for whole-cell patch-clamp recordings, as previously described (Zhou et al., 2010). Glass pipettes (resistance, 5-8 M $\Omega$ ) were pulled and filled with an internal solution containing the following (in $\mathrm{mM}$ ): 110 $\mathrm{Cs}^{+}$gluconate, 5 tetraethylammonium, $5 \mathrm{QX} 314,0.5 \mathrm{CaCl}_{2}, 5 \mathrm{BAPTA}$, 10 HEPES, $5 \mathrm{MgATP}$, and $0.33 \mathrm{GTP}-\mathrm{Tris}$, pH 7.3, $280 \mathrm{mOsm} / \mathrm{l}$. The input resistance was monitored, and the recording was discarded if it changed $>15 \%$. All electrophysiological signals were acquired using an Axon setup (Molecular Devices). Signals were sampled by pCLAMP 9.2 via an amplifier (Axopatch 200B) and an analog-to-digital converter (Digidata $1322 \mathrm{~A}$ ), filtered at $2-5 \mathrm{kHz}$, digitized at $10 \mathrm{kHz}$, and stored for off-line analysis. sEPSCs and mEPSCs were recorded at $-70 \mathrm{mV}$ in the presence of (-)bicuculline methiodide $(10 \mu \mathrm{M}), \mathrm{a}_{\mathrm{GABA}}$ receptor antagonist, and without or with tetrodotoxin $(1 \mu \mathrm{M})$, respectively.

Small-interfering RNA. The 19-nucleotide small-interfering RNA (siRNA) duplex was $5^{\prime}$-GCACAAGCUUUAUGUCAGA-3' for fRIM1 $\alpha$ and $5^{\prime}$-GAACAUUGAACACUUAAAC- 3 ' for Fbxl2. The missense nucleotide sequence was 5'-UGAUAUUACCCUGAAUAUG-3'. The missense or siRNA construct was intrathecally administered using a polyethyleneiminebased (10 $\mu \mathrm{l}$; Dharmacon) gene-delivery system into the dorsal subarachnoid space (L4-L5) of animals through the implanted catheter (daily for $4 \mathrm{~d})$.

Drug application. BC-1215 (a novel inhibitor of Fbxo3 activity; $100 \mathrm{nm,}$ $10 \mu \mathrm{l}$; Merck) was intrathecally administered by single bolus injection. $\omega$-Conotoxin GVIA $\left(\mathrm{Ca}_{\mathrm{V}} 2.2\right.$ blockers; 10,30 , and $100 \mathrm{pm} ; 10 \mu \mathrm{l}$; Bachem) was administered intrathecally by single bolus injection. A vehicle solution of equal volume to that of the tested agents was dispensed to serve as a control. The amplitude and frequency of sEPSCs were recorded in the presence of BC-1215 (300 nM), $\omega$-conotoxin GVIA (100 $\mathrm{nM}$ ), and nifedipine (an L-type calcium channel antagonist; $50 \mu \mathrm{M}$; Sigma-Aldrich).

Data analysis. The data in this study were analyzed using SigmaPlot 10.0 (Systat Software) or Prism 6.0 (GraphPad) and are expressed as the mean \pm SEM. Paired two-tailed Student's $t$ tests were used to compare the means between groups. One-way or two-way ANOVA was used to assess changes in values for serial measurements over time, and post hoc Tukey's tests were used to compare the means of groups. Significance was set at $p<0.05$. Mini Analysis 6.0 (Synaptosoft) was used to analyze the amplitude and frequency of sEPSCs and mEPSCs. The KolmogorovSmirnov test was used to compare the cumulative probability of sEPSCs frequency and amplitude.

\section{Results}

Nerve ligation enhances RIM1 $\alpha$ expression in the SPMs of the dorsal horn and provokes behavioral allodynia

To uncover the role of spinal RIM $1 \alpha$ in the development of neuropathic pain, we initially examined the amount of RIM $1 \alpha$ in the dorsal horn homogenate in response to neuropathic injury. Western blotting demonstrated that RIM1 $\alpha$ expression was significantly increased in the ipsilateral, but not the contralateral, dorsal horn on days $1,3,7,14$, and 21 after spinal nerve ligation (SNL; $0.40 \pm 0.04$, $0.45 \pm 0.02,0.57 \pm 0.03,0.52 \pm 0.03$, and $0.51 \pm 0.03$, respectively. $n=6$; Fig. $1 A$ ). Because RIM1 had previously been reported to be localized to the area of the SPMs (Wang et al., 2001), we used a subcellular fractionation to examine RIM1 $\alpha$ expression in the SPMs of the dorsal horn following nerve ligation. The abundance of RIM $1 \alpha$ in the SPMs of the ipsilateral, but not the contralateral, dorsal horn increased after SNL $(0.59 \pm 0.03,0.61 \pm 0.03,0.81 \pm 0.03$, $0.78 \pm 0.03$, and $0.80 \pm 0.02$, respectively. $n=6)$ in parallel with that in the total homogenate (Fig. $1 B$ ). Moreover, the SNL-enhanced RIM1 $\alpha$-expression levels in the homogenate and SPM were both temporally aligned with the SNL-induced tactile allodynia (2.77 \pm $0.44,1.62 \pm 0.42,0.30 \pm 0.07,0.61 \pm 0.14$, and $0.51 \pm 0.14 \mathrm{~g}$, respectively. $n=7$; Fig. $1 C$ ). Together, these data suggest that neu- 
A

Sham 7D

SNL 7D
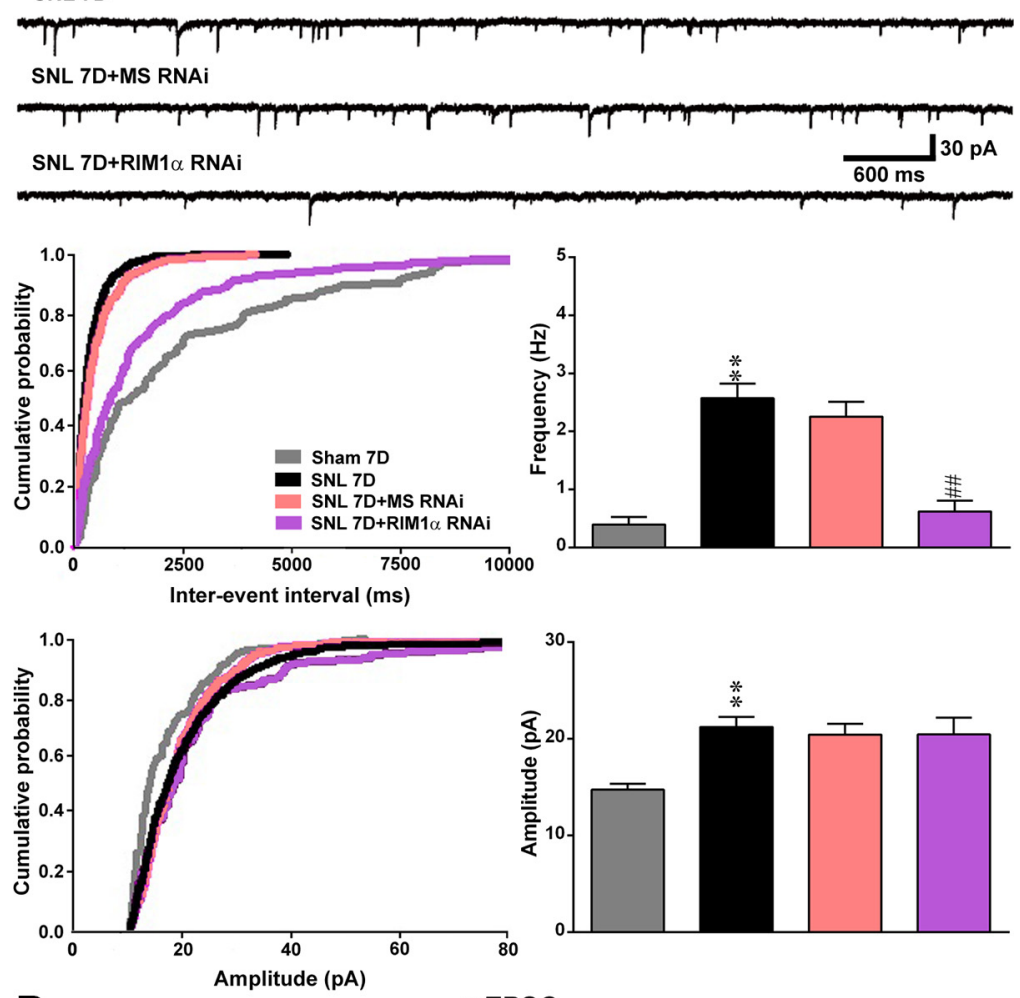

B

Sham 7D

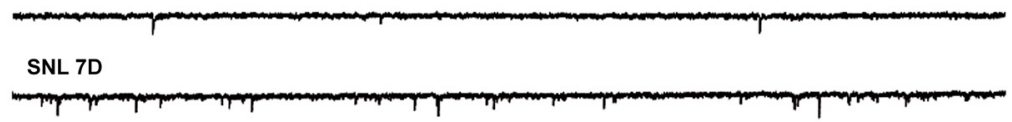

SNL 7D+MS RNAi
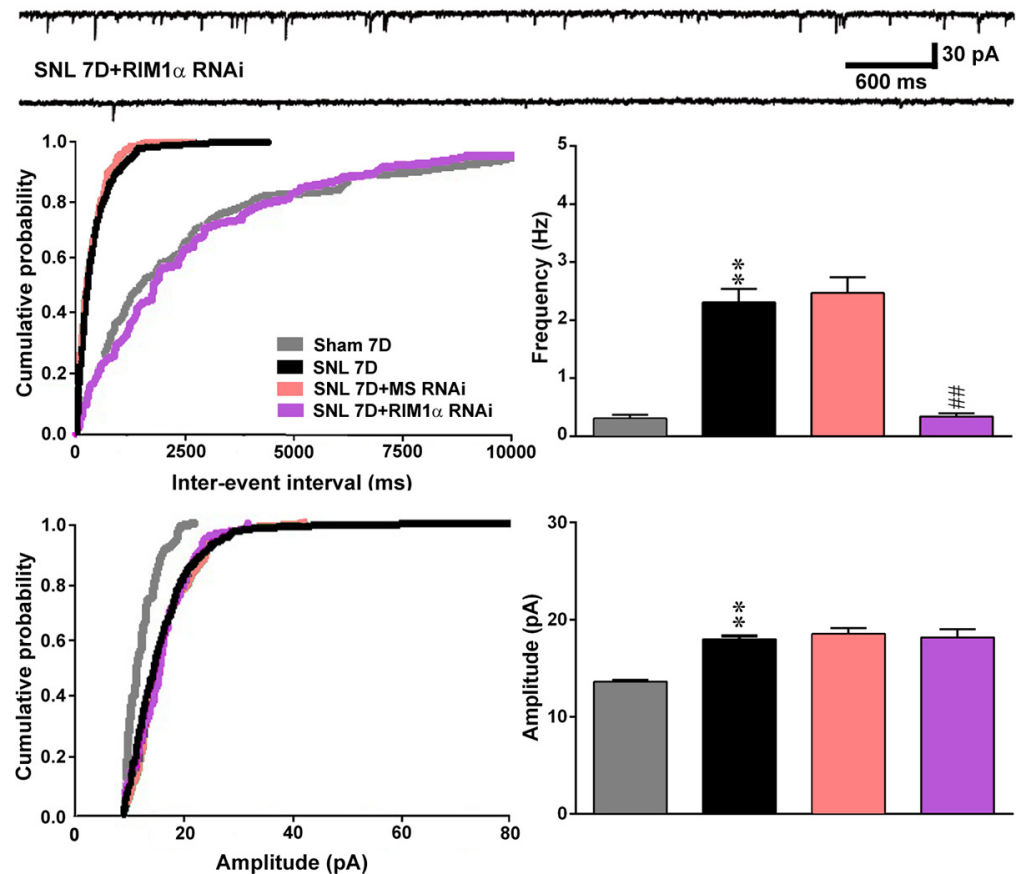

Figure 3. Knockdown of spinal RIM1 $\alpha$ expression decreases the SNL-enhanced frequency of sEPSCs and mEPSCs in ipsilatera dorsal horn neurons. $\boldsymbol{A}$, Top, Representative traces of $s E P S C$ s recorded in the ipsilateral dorsal horn neuron of spinal slices isolated from animals receiving the sham-operation (Sham 7D), SNL (SNL 7D), SNL treated with missense siRNA (SNL 7D+MS RNAi), and ropathic injury induces nociceptive hypersensitivity associated with enhanced RIM1 $\alpha$ expression in the SPM of the ipsilateral dorsal horn.

\section{Knocking down of spinal RIM1 $\alpha$ expression ameliorates \\ SNL-induced allodynia}

To provide a genetic basis for the role of spinal RIM $1 \alpha$ in the development of neuropathic pain, we generated rats in which spinal RIM1 $\alpha$ expression was focally knocked down through the daily intrathecal administration of antisense siRNA specifically targeting the RIM $1 \alpha$ mRNA. First, Western blotting demonstrated a dose-dependent decrease in the abundance of RIM $1 \alpha$ expression in the dorsal horn samples of naive rats following administration with RIM1 $\alpha$ mRNAtargeting siRNA $(1,3$, and $5 \mu \mathrm{g} ; 10 \mu \mathrm{l}$; once daily for $4 \mathrm{~d}$; $0.18 \pm 0.02,0.07 \pm$ $0.02,0.04 \pm 0.01$, respectively; $n=6$; Fig. $2 A$ ) but not with missense siRNA ( $5 \mu \mathrm{g}$, $10 \mu \mathrm{l}$ ), polyethylenimine (a transfection reagent, $10 \mu \mathrm{l}$ ), or intrathecal catheter implantation alone. In contrast, the expression of spinal RIM2 expression remained at a relatively constant level across groups (Fig. 2B). This finding suggests that spinal RIM $1 \alpha$ expression was specifically knocked down by the administration with antisense siRNA. Subsequent rotarod

\section{$\leftarrow$}

SNL treated with RIM1 $\alpha$ mRNA-targeting antisense siRNA (SNL 7D + RIM1 $\alpha$ RNAi). Bottom left, Cumulative probability histograms of the interevent interval and the amplitude of SEPSCs recorded from all groups. Note that the interevent interval of sEPSCs was significantly shorter and the amplitude was larger in the SNL group than in the sham-operated group ( $p<0.01$, Kolmogorov-Smirnov test). RIM1 $\alpha$ mRNAtargeting antisense siRNA prolonged interevent intervals of SEPSCS compared with the SNL group ( $p<0.01$, Kolmogorov-Smirnov test). Bottom right, Average frequency $\left(F_{(3,16)}=27.25\right.$, one-way ANOVA, $p<0.001$, posthoc Tukey's tests; ${ }^{* *} p<0.01$ vs Sham 7D; \#\#p $<0.01$ vs SNL) and amplitude $\left(F_{(3,16)}=6.44\right.$, one-way ANOVA, $p<0.01$, post hoc Tukey's tests; ${ }^{* *} p<0.01$ vs Sham 7D) of sEPS(s in animals receiving the sham operation, $S N L, S N L$ treated with missense siRNA, and SNL treated with RIM1 $\alpha$ mRNA-targeting antisense siRNA. $\boldsymbol{B}$, Top, Representative traces of $\mathrm{mEPSC}$ (s recorded in the ipsilateral dorsal horn neurons of spinal slices isolated from groups. Bottom left, Cumulative probability histograms of the interevent interval and the amplitude of mEPSCs. The interevent interval of $\mathrm{mEPSC}$ s was significantly shorter and the amplitude was larger in SNL animals than in the shamoperated animals ( $p<0.01$, Kolmogorov-Smirnov test). RIM1 $\alpha$ mRNA-targeting antisense siRNA prolonged interevent intervals of mEPSCs compared with the SNL group ( $p<$ 0.01 , Kolmogorov-Smirnov test). Bottom right, Average frequency $\left(F_{(3,17)}=38.91\right.$, one-way ANOVA, $p<0.001$, post hoc Tukey's tests. ${ }^{* *} p<0.01$ vs Sham 7D; \#\#p $<0.01$ vs SNL) and amplitude $\left(F_{(3,17)}=18.13\right.$, one-way ANOVA, $p<0.01$, post hoc Tukey's tests; ${ }^{* *} p<0.01$ vs Sham 7D) of mEPS(S. 
A
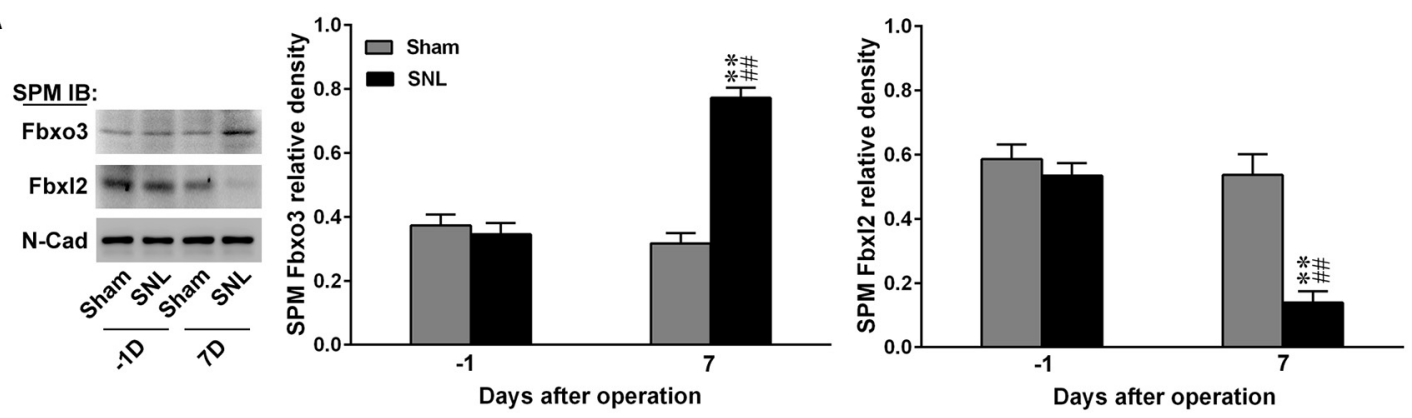

B
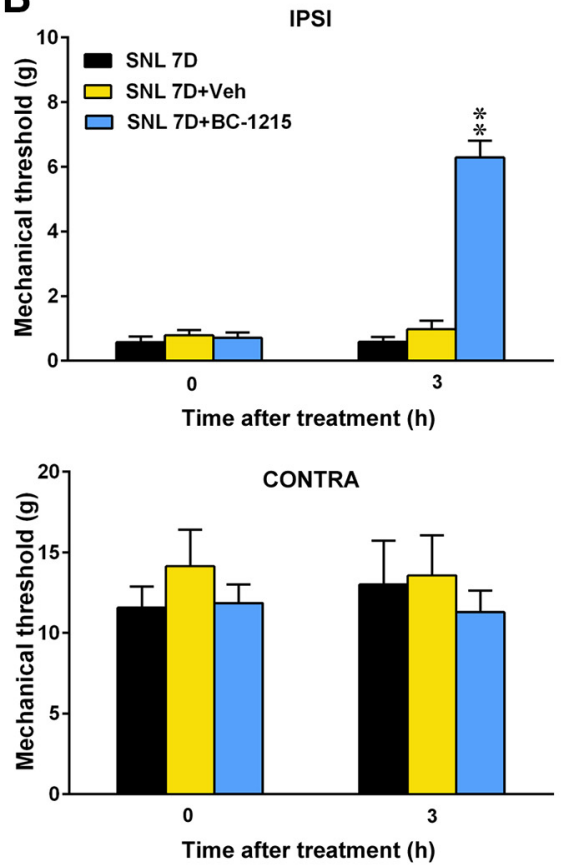

D
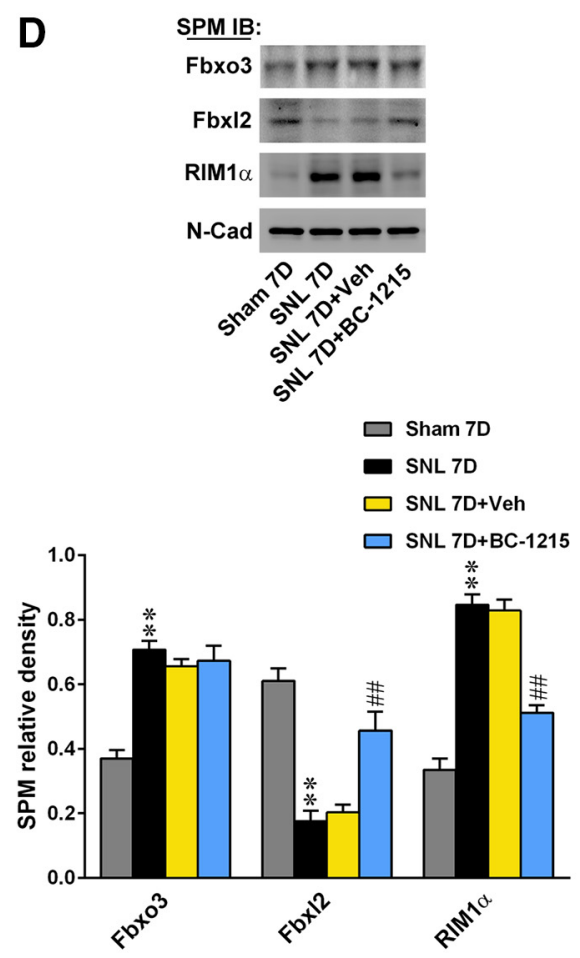

C
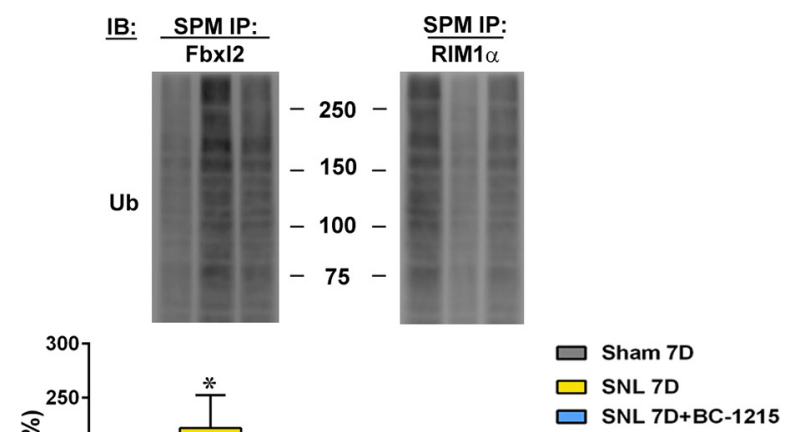

E

SPM IB:

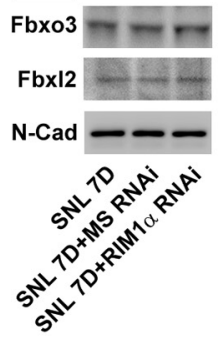

$\square$ SNL 7D

$\square$ SNL 7D+MS RNAi

$\square$ SNL 7D+RIM1 $\alpha$ RNAi

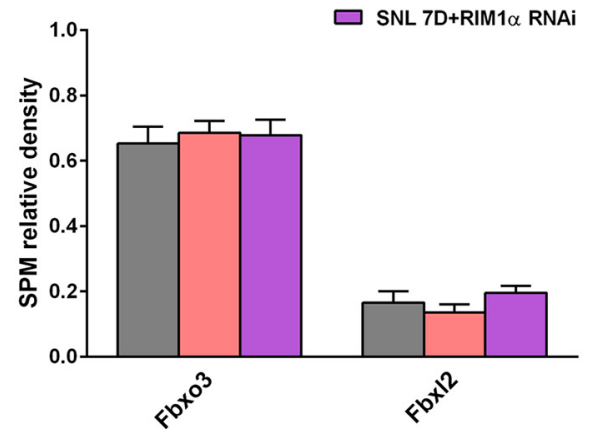


analysis showed no significant differences in the motor performance among the naive and polyethylenimine-treated $(10 \mu \mathrm{l})$, missense siRNA-treated $(5 \mu \mathrm{g}, 10 \mu \mathrm{l})$, or RIM1 $\alpha$ mRNAtargeting siRNA-treated ( $5 \mu \mathrm{g}, 10 \mu \mathrm{l}$ ) groups (Fig. $2 C$ ), suggesting that neither these procedures nor the spinal $\operatorname{RIM} 1 \alpha$ knockdown led to motor deficits in rats. Although none of the treatments exhibited any effects on the withdrawal threshold of the sham-operated animals (Fig. 2D), the results of the von Frey test showed that the daily administration of RIM $1 \alpha$ mRNAtargeting siRNA $(5 \mu \mathrm{g}, 10 \mu \mathrm{l})$ partially ameliorated SNL-induced behavioral allodynia, as evidenced by a significant increase in the withdrawal threshold on days 5 and 7 after the operation $(5.14 \pm$ 0.73 and $6.00 \pm 0.87$ g, respectively; $n=7$; Fig. $2 E)$. Moreover, administration with RIM1 $\alpha$ mRNA-targeting siRNA ( $5 \mu \mathrm{g}, 10$ $\mu \mathrm{l})$ significantly decreased the RIM $1 \alpha$ expression in both the total homogenate (from $0.27 \pm 0.02$ to $0.04 \pm 0.01 \mathrm{~g}$ and from $0.65 \pm 0.02$ to $0.34 \pm 0.05$, respectively; $n=6$; Fig. $2 F$ ) and SPM (from $0.37 \pm 0.02$ to $0.06 \pm 0.01 \mathrm{~g}$ and from $0.83 \pm 0.03$ to $0.43 \pm$ 0.03 , respectively; $n=6$; Fig. $2 G$ ) of the ipsilateral dorsal horn samples dissected at postoperative day 7 from the sham-operated and SNL-operated groups. Collectively, the data obtained from the knockdown animals suggested that the increase in RIM1 $\alpha$ in the SPM of the ipsilateral dorsal horn contributes to the SNLassociated nociceptive hypersensitivity.

\section{RIM1 $\alpha$ in pre-SPMs of the dorsal horn contributes to SNL-induced allodynia}

The above experimental results showed that RIM $1 \alpha$ expression in the SPM of the dorsal horn was increased after SNL. However, the precise location of the SNL-enhanced RIM1 $\alpha$, i.e., at the presynaptic or postsynaptic membrane, is still uncertain. Therefore, to further identify the involvement of presynaptic and postsynaptic RIM1 $\alpha$ in SNL-induced allodynia, we assessed sEPSCs, which are action potential-dependent presynaptic events, by electrophysiological recording in acute spinal slices dissected

\footnotetext{
$\leftarrow$

Figure 4. BC-1215 relieves allodynia by promoting Fbx|2-dependent RIM1 $\alpha$ ubiquitination. $A$, Representative Western blotting and statistical analysis (normalized to $\mathrm{N}$-cadherin) demonstrating that SNL increased Fbxo3 but decreased FbxI2 in the SPMs of the ipsilateral dorsal horn at day 7 after the operation. SPM IB, SPM immunoblotting. Student's $t$ test. ${ }^{* *} p<0.01$ vs Sham 7D;\#\# $<0.01$ vs SNL Day $-1 ; n=6 . B$, At postoperative day 7, the intrathecal administration with BC-1215 (SNL 7D + BC-1215; $100 \mathrm{~nm} ; 10 \mu \mathrm{l})$, but not the vehicle solution (SNL 7D + Veh, $10 \mu \mathrm{l})$, increased the withdrawal threshold of the ipsilateral (IPSI) hindpaw in the SNL rat $3 \mathrm{~h}$ after injection. Nevertheless, both of these treatments failed to affect the contralateral (CONTRA) hindpaw at the same time point ( $100 \mathrm{~nm}$; von Frey test). IPSI $0 \mathrm{~h}$, one-way ANOVA, $F_{(2,18)}=0.37, p=0.70$, post hoc Tukey's tests; IPSI 3 h, one-way ANOVA, $F_{(2,18)}=84.44, p<$ 0.001 , post hoc Tukey's tests; CONTRA $0 \mathrm{~h}$, one-way ANOVA, $F_{(2,18)}=0.73, p=0.50$, post hoc Tukey's tests. CONTRA $3 \mathrm{~h}$, one-way ANOVA, $F_{(2,18)}=0.27, p=0.76$, post hoc Tukey's tests; ${ }^{* *} p<0.01$ vs SNL 7D 3 h. $n=7$. C, On day 7 after the operation, the SNL increased Fbxl2 but decreased RIM1 $\alpha$ ubiquitination in the SPMs of the ipsilateral dorsal horn (SNL 7D), and this was markedly reversed by spinal injection of BC-1215 (SNL 7D + BC-1215; $100 \mathrm{nM}, 10 \mu \mathrm{l})$. SPM IP, SPM immunoprecipitation; IB, Immunoblotting. Fbx|2, one-way ANOVA, $F_{(2,12)}=6.77, p=$ 0.010 , post hoc Tukey's tests; Fbx03, one-way ANOVA, $F_{(2,12)}=7.40, p=0.008$, post hoc Tukey's tests; ${ }^{*} p<0.05$ vs Sham 7D; $\# p<0.05$ vs SNL 7D; $n=5$. D, Without affecting the abundance of Fbx03, BC-1215 (SNL 7D + BC-1215; $100 \mathrm{~nm}, 10 \mu \mathrm{l}$ ) reversed the SNL-decreased FbxI2 and SNL-increased RIM1 $\alpha$ expression in the SPMs of the ipsilateral dorsal horn. Fbxo3, one-way ANOVA, $F_{(3,20)}=23.78, p<0.001$, post hoc Tukey's tests; Fbxl2, one-way ANOVA, $F_{(3,20)}=26.18, p<0.001$, post hoc Tukey's tests; RIM1 $\alpha$, one-way ANOVA, $F_{(3,20)}=63.67$, $p<0.001$, posthoc Tukey's tests; ${ }^{* *} p<0.01$ vs Sham 7D; \#\#p $<0.01$ vs SNL 7D; $n=6 . E$, The administration with neither RIM1 $\alpha$ mRNA-targeting siRNA (SNL 7D + RIM1 $\alpha$ RNAi; $5 \mu \mathrm{g}, 10$ $\mu$ l) nor missense siRNA (SNL 7D + MS RNAi; $5 \mu \mathrm{g}, 10 \mu \mathrm{l}$ ) affected spinal Fbx03 or FbxI2 expression in the SPM of the ipsilateral dorsal horn on day 7 after SNL. Fbx03, one-way ANOVA, $F_{(2,15)}=0.15, p=0.87$, post hoc Tukey's tests; Fbxl2, one-way ANOVA, $F_{(2,15)}=1.19, p=$ 0.33 , post hoc Tukey's tests; $n=6$.
}

from different groups. In SNL animals, both the frequency and amplitude of the sEPSCs were significantly increased in the ipsilateral spinal dorsal neurons on postoperative day 7 compared with the sham-operated group (frequency: Sham, $0.39 \pm 0.12$, $n=5$; SNL, $2.57 \pm 0.24, n=5$; amplitude: Sham, $14.73 \pm 0.59$, $n=5$; SNL, $21.21 \pm 1.02, n=5$; Fig. $3 A$ ). Notably, spinal administration with RIM $1 \alpha$ mRNA-targeting siRNA (5 $\mu \mathrm{g}, 10 \mu \mathrm{l})$ significantly decreased the SNL-enhanced frequency but exhibited no effect on the amplitude of the sEPSCs (frequency: $0.62 \pm 0.18$; amplitude: $20.44 \pm 1.72, n=5$; Fig. $3 A$ ). Conversely, neither the frequency nor amplitude of the sEPSCs was affected in dorsal neurons dissected from rats receiving intrathecal application of missense siRNA ( $5 \mu \mathrm{g}, 10 \mu \mathrm{l}$; frequency: $2.25 \pm 0.25$; amplitude: $20.43 \pm 1.10, n=5)$. To rule out the possibility that spinal RIM $1 \alpha$ knockdown-associated decrement in sEPSC frequency in the SNL animal could be attributed to reducing the number of action potentials in axon transmission, we next recorded mEPSCs, a spontaneous synaptic activity independent of presynaptic action potential. We observed that while the enhanced amplitude was not affected (amplitude: SNL, $18.0 \pm 0.33, n=6$; SNL+RIM1 $\alpha$ RNAi, $18.18 \pm 0.84, n=5$ ), the increased frequency of mEPSCs was significantly reduced in RIM $1 \alpha$ knockdown animals (SNL, $2.30 \pm 0.23, n=6$; SNL+RIM1 $\alpha$ RNAi, $0.34 \pm 0.06, n=5$; Fig. $3 B$ ). This result, similar to the result of sEPSCs, suggests that focal knockdown of spinal RIM $1 \alpha$ predominantly decreased presynaptic release but has minor axonal effects. Collectively, these results suggest that the SNL induced allodynia and enhanced sEPSC frequency through the RIM $1 \alpha$ dependent mechanism in the presynaptic membrane.

\section{BC-1215 relieves allodynia through activation of Fbxl2-} dependent RIM1 $\alpha$ ubiquitination in SPMs of the dorsal horn At presynaptic terminals in vitro, activity-dependent synaptic function is regulated by E3-ubiquitin ligase (E3)-mediated protein ubiquitination (Yi and Ehlers, 2005). A recent study has found that Fbxl2, a synapse-localized E3 ubiquitin ligase, directly binds to and ubiquitinates RIM1 to regulate synaptic vesicle release (Yao et al., 2007). As our previous study showed that Fbxo3, another E3-ubiquitin ligase subunit, contributes to the development of neuropathic pain by destabilizing Fbxl2 (Lin et al., 2015a), we speculated that presynaptic RIM1 $\alpha$ contributes to neuropathic pain through Fbxo3-abating Fbxl2-dependent RIM $1 \alpha$ ubiquitination in the SPM of the dorsal horn. To address this issue, we first assayed spinal Fbxo3 and Fbxl2 expression in the SPM following nerve ligation. Western blotting showed that SNL significantly increased Fbxo3 abundance $(0.77 \pm 0.03, n=$ 6) but decreased Fbxl 2 abundance $(0.13 \pm 0.03, n=6)$ in the SPM of the ipsilateral dorsal horn sample on postoperative day 7 (Fig. 4A). Next, we examined whether the intrathecal application of BC-1215, a novel activity inhibitor of Fbxo3, could modify SNL-induced behavioral allodynia. The results of the von Frey test demonstrated that a single injection bolus of BC-1215 (100 $\mathrm{nm}, 10 \mu \mathrm{l})$, but not the vehicle solution $(10 \mu \mathrm{l})$, significantly ameliorated SNL-induced behavioral allodynia by $3 \mathrm{~h}$ after injection (from $0.58 \pm 0.15$ to $6.28 \pm 0.52 \mathrm{~g}, n=7$ ), a time point at which BC-1215 was shown to exhibit maximal effect on withdrawal threshold of SNL rats (Lin et al., 2015b). Nevertheless, application of neither the vehicle solution nor BC-1215 (100 nM, $10 \mu \mathrm{l})$ affected the withdrawal threshold of the contralateral hindpaw at this time point (Fig. $4 B$ ). Based on these results, we further examined whether BC-1215 exerted its analgesic effect by attenuating Fbxo3-dependent Fbxl2 ubiquitination, resulting in enhanced RIM $1 \alpha$ ubiquitination and degradation in the SPM of 
A

IB:

FbxI2

GAPDH

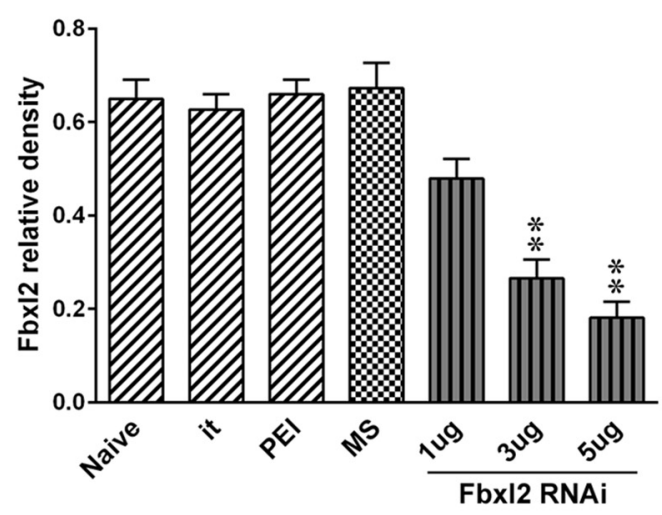

C
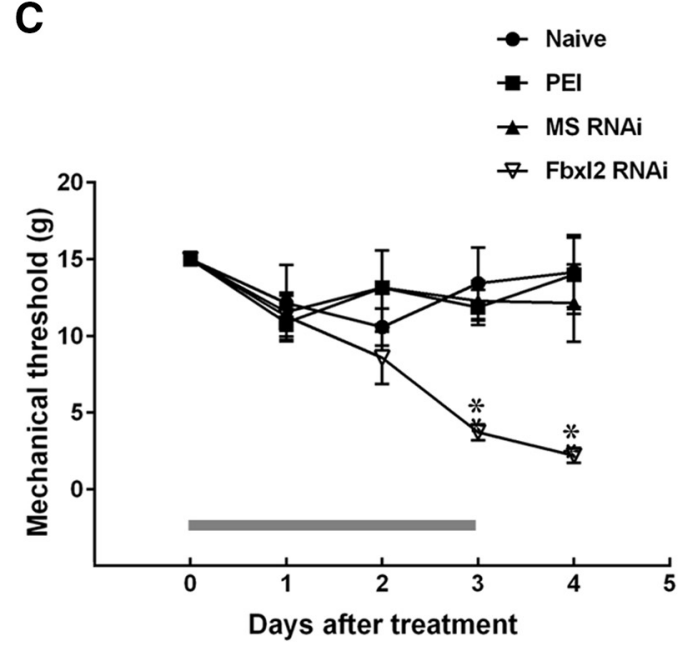

E

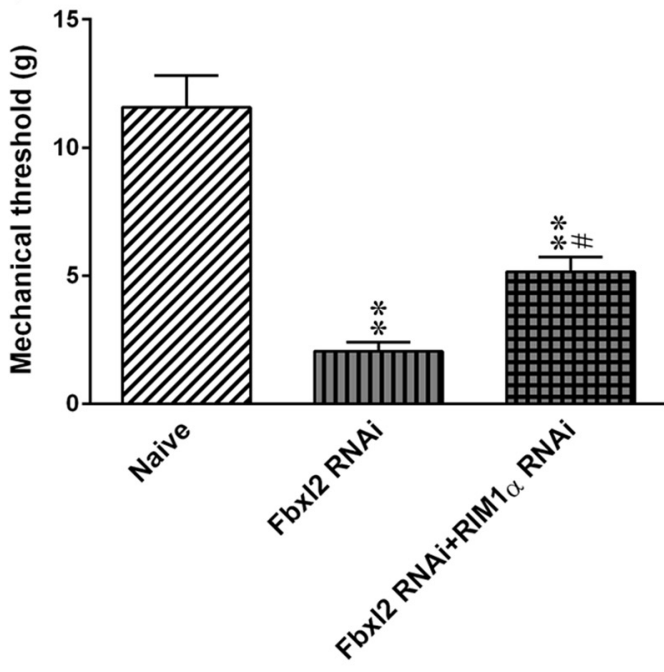

B

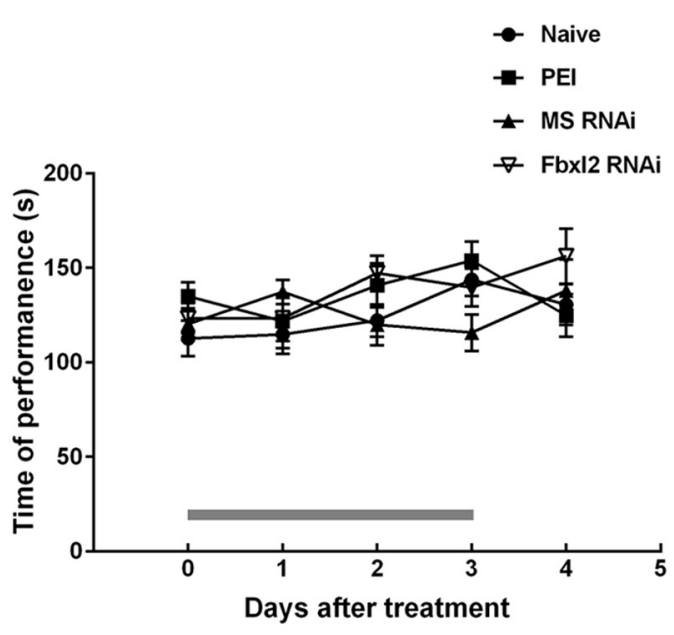

D

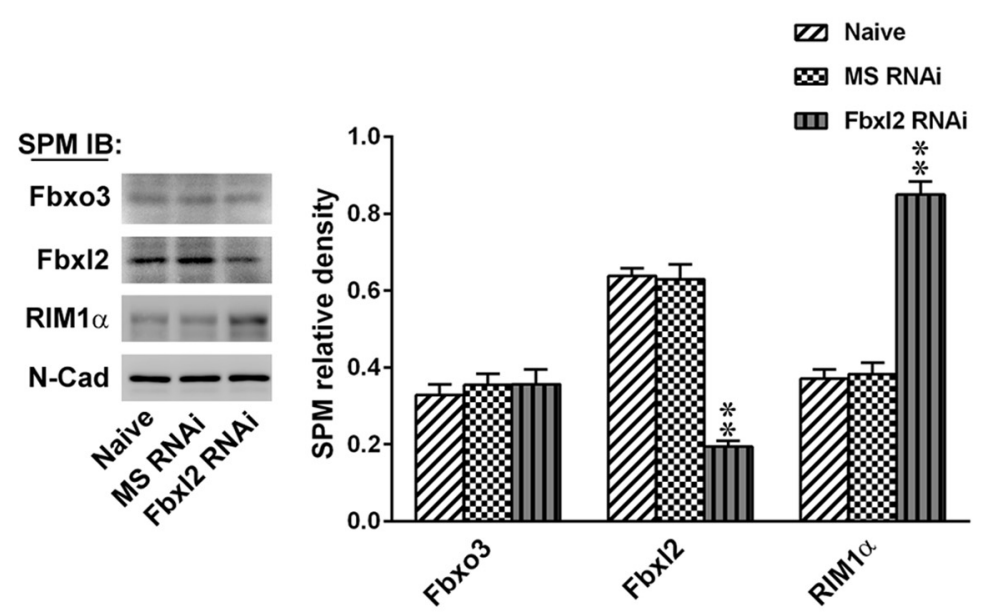

F

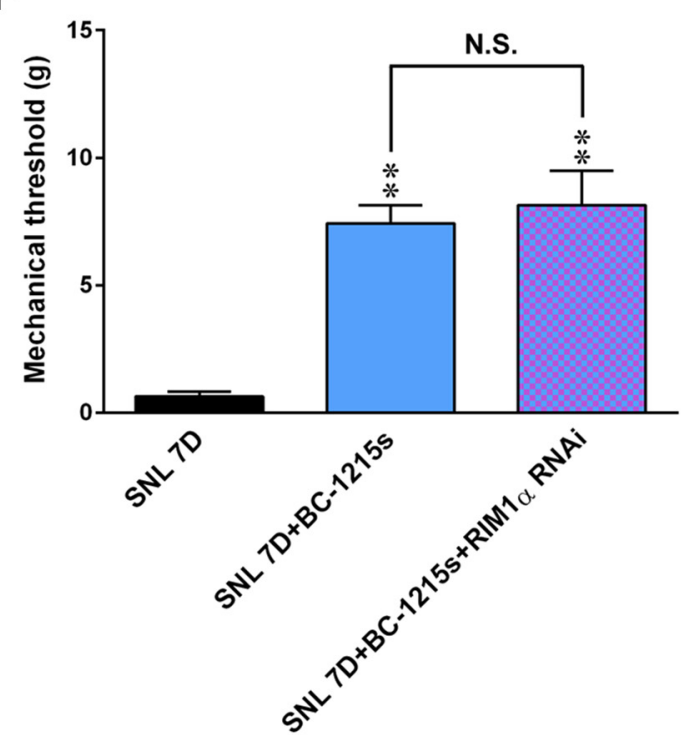

Figure 5. Knockdown of spinal FbxI2 expression provokes behavioral allodynia by increasing RIM1 $\alpha$ expression without affecting the abundance of Fbxo3 in the ipsilateral dorsal horn. $A$, Representative Western blotting and statistical analysis (normalized to GAPDH) demonstrating that intrathecal administration of Fbxl2 mRNA-targeting siRNA (Fbxl2 RNAi; 1,3 , and $5 \mu \mathrm{g}$; $10 \mu \mathrm{l}$; once daily for $4 \mathrm{~d}$ ), but not missense siRNA (MS RNAi; $5 \mu \mathrm{g}, 10 \mu \mathrm{l}$ ) or polyethylenimine (a transfection reagent, PEl; $10 \mu \mathrm{l}$ ), dose-dependently decreased spinal FbxI2 expression of naive rat. it, Implantation of an intrathecal catheter; IB, immunoblotting. One-way ANOVA, post hoc Tukey's test, $F_{(6,35)}=26.07, p<0.001 ;{ }^{*} p<0.05$, ${ }^{* *} p<0.01$ (Figure legend continues.) 
the dorsal horn. At day 7 postoperation, SNL predictably increased Fbxl2 ubiquitination in the SPM of the ipsilateral dorsal horn (from $91.00 \pm 19.42$ to $222.00 \pm 30.52 \%, n=5$ ), which was markedly reversed by an intrathecal BC-1215 injection (100 nM, $10 \mu \mathrm{l} ; 117.60 \pm 28.57 \%, n=5)$. Moreover, administrating BC$1215(100 \mathrm{nM}, 10 \mu \mathrm{l})$ to SNL animals with through the intrathecal route robustly increased RIM $1 \alpha$ ubiquitination in the SPM of the ipsilateral dorsal horn $(96.80 \pm 22.96 \%, n=5)$ compared with the untreated animals $(22.80 \pm 11.14 \%, n=5$; Fig. $4 C)$. In addition, we observed that BC-1215 ( $100 \mathrm{nM}, 10 \mu \mathrm{l})$ reversed not only SNL-enhanced RIM $1 \alpha$ expression (from $0.84 \pm 0.03$ to $0.51 \pm$ $0.02, n=6)$ but also reversed SNL-decreased Fbxl2 expression (from $0.17 \pm 0.03$ to $0.45 \pm 0.05, n=6$ ) without affecting the abundance of Fbxo3 in the SPM of the ipsilateral dorsal horn on day 7 after SNL (Fig. 4D). Importantly, the administration of either RIM $1 \alpha$ mRNA-targeting siRNA ( $5 \mu \mathrm{g}, 10 \mu$ l, daily for $4 \mathrm{~d}$ ) or missense siRNA $(10 \mu \mathrm{l})$ did not affect the Fbxo3 or Fbxl2 expression in the SPM of the ipsilateral dorsal horn on day 7 after SNL (Fig. 4E), implying that spinal Fbxo3 and Fbxl2 in the SPM act as upstream proteins of RIM $1 \alpha$ in neuropathic pain development. Collectively, these results suggest that SNL-associated Fbxo3 expression participates in the development of neuropathic pain by ubiquitinating Fbxl2, thereby decreasing the Fbxl2dependent RIM $1 \alpha$ ubiquitination in the SPM of the ipsilateral dorsal horn.

\section{Knocking down spinal Fbxl2 expression provokes behavioral allodynia by increasing RIM1 $\alpha$ expression without affecting the abundance of Fbxo3}

To further clarify the upstream and/or downstream relationships between $\mathrm{Fbxo} 3$ and Fbxl2, we next generated rats in which spinal Fbxl2 expression was focally knocked down through daily intrathecal administration with antisense siRNA specifically targeting Fbxl2 mRNA. Western blotting demonstrated a dose-dependent decrease in the abundance of Fbxl2 expression in the dorsal horn samples of naive rats receiving an intrathecal injection of Fbxl2 mRNA-targeting siRNA $(1,3$, and $5 \mu \mathrm{g} ; 10 \mu \mathrm{l}$; once daily for $4 \mathrm{~d}$; $0.47 \pm 0.04,0.26 \pm 0.04,0.18 \pm 0.03$, respectively; $n=6$; Fig. $5 A$ ), indicating that spinal Fbxl2 expression was knocked down

\section{$\leftarrow$}

(Figurelegend continued.) $\quad$ vs Naïve; $n=6 . B, C$, The focal knockdown of spinal Fbx|2 (Fbxl2 RNAi; $5 \mu \mathrm{g}, 10 \mu \mathrm{ll}$ ) resulted in no significant differences in the time of rotarod performance ( $\boldsymbol{C}$, rotarod test) but decreased the withdrawal threshold on days 3 and 4 following the start of treatment $(\boldsymbol{D}$, von Frey test). The gray bar at the bottom indicates the duration of intrathecal administration. Rotarod performance, two-way ANOVA with repeated measures over time, treatment, $F_{(3,24)}=1.79, p=0.17$; time, $F_{(4,96)}=1.84, p=0.13$; treatment $\times$ time, $F_{(12,96)}=1.31, p=0.22$. von Frey test, two-way ANOVA with repeated measures overtime, treatment, $F_{(3,24)}=6.62, p=0.002$; time, $F_{(4,96)}=7.56$, $p<0.001$; treatment $\times$ time, $F_{(12,96)}=4.20, p<0.001$; ${ }^{* *} p<0.01$ vs Naïve; $n=7$. D, Without affecting Fbxo3 expression, focal knockdown of spinal Fbxl2 expression (Fbxl2 RNAi; $5 \mu \mathrm{g}, 10 \mu \mathrm{l}$ ) decreased the abundance of Fbxl2 and increased the abundance of RIM1 $\alpha$ in the SPMs of the ipsilateral dorsal horn.SPM IB,SPMimmunoblotting. Fbxo3, one-way ANOVA, $F_{(2,15)}=0.24, p=0.79$, post hoc Tukey's tests; FbxI2, one-way ANOVA, $F_{(2,15)}=92.50, p<0.001$, posthoc Tukey's tests; RIM1 $\alpha$, one-way ANOVA, $F_{(2,15)}=85.33, p<0.001$, post hoc Tukey's tests; ${ }^{* *} p<0.01$ vs Naïve; $n=6$. $\boldsymbol{E}$, When compared with the decreased withdrawal threshold in the Fbxl2 knocked-down animals (Fbxl2 RNAi; $5 \mu \mathrm{g}, 10 \mu$ l), additional knockdown of RIM1 $\alpha$ partially reversed the decreased withdrawal threshold of the ipsilateral hindpaw (Fbxl2 RNAi + RIM1 $\alpha$ RNAi; both $5 \mu \mathrm{g}, 10 \mu$ l). One-way ANOVA, $F_{(2,18)}=34.56, p<0.001$, post hoc Tukey's tests; ${ }^{* *} p<0.01$ vs Naiive; $\# p<0.01$ vs Fbxl2 $\mathrm{RNAi} ; n=7$. $F$, When compared with daily spinal injection of $\mathrm{BC}-1215$ significantly increased the withdrawal threshold of the ipsilateral hindpaw (SNL 7D + BC-1215s; $100 \mathrm{~nm}, 10 \mu$; daily for $4 \mathrm{dfrom}$ day 3 to day 6 after SNL) additional administration with RIM1 $\alpha$ mRNA-targeting siRNA (SNL 7D + BC$1215+$ RIM1 $\alpha$ RNAi; $5 \mu \mathrm{g}, 10 \mu$; daily for $4 \mathrm{~d}$ from day 3 to day 6 after SNL) did not further increase the withdrawal threshold ( $100 \mathrm{~nm} ; 10 \mu \mathrm{l}$; von Freytest). One-way ANOVA, $F_{(2,18)}=21.63, p<0.001$, post hoc Tukey's tests; ${ }^{* *} p<0.01$ vs SNL 7D; $n=7$. by specific siRNA. Subsequent rotarod analysis showed no significant difference in the motor performance among the naive, polyethylenimine-treated ( $10 \mu \mathrm{l})$, missense siRNA-treated ( $5 \mu \mathrm{g}$, $10 \mu \mathrm{l})$, and Fbxl2 mRNA-targeting siRNA-treated (5 $\mu \mathrm{g}, 10 \mu \mathrm{l}$ ) groups (Fig. $5 B$ ), suggesting that neither these procedures nor the spinal Fbxl2 knockdown led to motor deficits in rats. Notably, the focal knockdown of spinal Fbxl2 expression ( $5 \mu \mathrm{g}, 10 \mu \mathrm{l})$ resulted in a decreased withdrawal threshold compared with the naive rats on days 3 and 4 after treatment $(3.71 \pm 0.52$ and $2.20 \pm 0.48 \mathrm{~g}$; $n=7$; Fig. $5 C$ ), suggesting that the decrease in spinal Fbxl2 expression is an essential factor in the development of SNLassociated allodynia. Moreover, administration with Fbxl2 mRNA-targeting siRNA ( $5 \mu \mathrm{g}, 10 \mu \mathrm{l}$ ) not only decreased Fbxl2 expression (from $0.63 \pm 0.01$ to $0.19 \pm 0.01, n=6$ ) but also increased RIM $1 \alpha$ expression (from $0.38 \pm 0.03$ to $0.84 \pm 0.03$, $n=6$ ) without affecting the abundance of Fbxo3 expression in the SPM of the ipsilateral dorsal horn on day 7 after SNL (Fig. $5 D)$. We next clarify whether the RIM $1 \alpha$ plays as one of the downstream effectors of Fbxo3/Fbxl2 cascade in the neuropathic injury-induced allodynia. First, we examined whether knockdown of spinal RIM1 $\alpha$ expression occludes the effect of Fbxl2 siRNA. In naive animals, trim-down of spinal RIM $1 \alpha$ expression (5 $\mu \mathrm{g}, 10 \mu \mathrm{l}$ ) partially reversed the decrement of withdrawal threshold caused by administration with Fbxl2 mRNA-targeting antisense siRNA ( $5 \mu \mathrm{g}, 10 \mu \mathrm{l}$; from $2.05 \pm 0.35$ to $5.14 \pm 0.59 \mathrm{~g}$, $n=7$; Fig. $5 E$ ). In addition, focal knockdown of spinal RIM $1 \alpha$ expression $(5 \mu \mathrm{g}, 10 \mu \mathrm{l})$ exhibited no additional analgesic effect on BC-1215 (100 nM, $10 \mu \mathrm{l})$ for the withdraw threshold has no significant difference between daily BC-1215-treated SNL rats with and without daily RIM $1 \alpha$ mRNA-targeting antisense siRNA $(7.42 \pm 0.71$ and $8.14 \pm 1.35$ g, respectively; $n=7$; Fig. $5 F)$. These data further support the proposal that spinal Fbxl2 functions as a downstream molecule of Fbxo3 and subsequently activates RIM $1 \alpha$ in the SPM of the ipsilateral dorsal horn to underlie the development of neuropathic pain.

\section{BC-1215 reduces SNL-enhanced frequency and amplitude of sEPSCs in spinal ipsilateral slices}

A previous study demonstrated that SNL-enhanced spinal Fbxo3 expression underlies the development of neuropathic pain by affecting postsynaptic components of the spinal neural circuitry (Lin et al., 2015b). Moreover, the above results showed that pharmacological antagonism of spinal Fbxo3 alleviated SNL-induced allodynia by modifying presynaptic RIM $1 \alpha$-dependent machinery. Nevertheless, whether spinal Fbxo3 could also contribute to neuropathic pain mechanisms by influencing the presynaptic components of neural transmission in the dorsal horn is not clear. Therefore, we investigated the impact of BC-1215 on the SNL-enhanced frequency/amplitude of sEPSCs recorded from dorsal horn neurons in spinal slices dissected from SNL animals. Interestingly, incubation of slices with BC-1215 (300 nM) for $3 \mathrm{~h}$ significantly reversed the SNL-induced enhanced frequency and amplitude of sEPSCs in dorsal horn neurons (frequency: SNL, $2.40 \pm 0.23, n=6$; SNL+BC-1215, $0.33 \pm 0.13, n=4$; amplitude: SNL, $21.17 \pm 1.12, n=6$; SNL+BC-1215, $14.16 \pm 0.89$, $n=4$; Fig. $6 A$ ), indicating that Fbxo3 contributes to the SNLinduced allodynia through not only postsynaptic but also presynaptic sites of the dorsal horn.

\section{SNL provokes enhancement of spinal presynaptic RIM1 $\alpha$ expression associated with $\mathrm{Ca}_{\mathrm{v}} 2.2$}

$\mathrm{RIM}, \mathrm{a} \mathrm{Ca}^{2+}$-dependent synaptic vesicle priming factor, is essential for vesicle priming and the subsequent recruitment of the nearby 

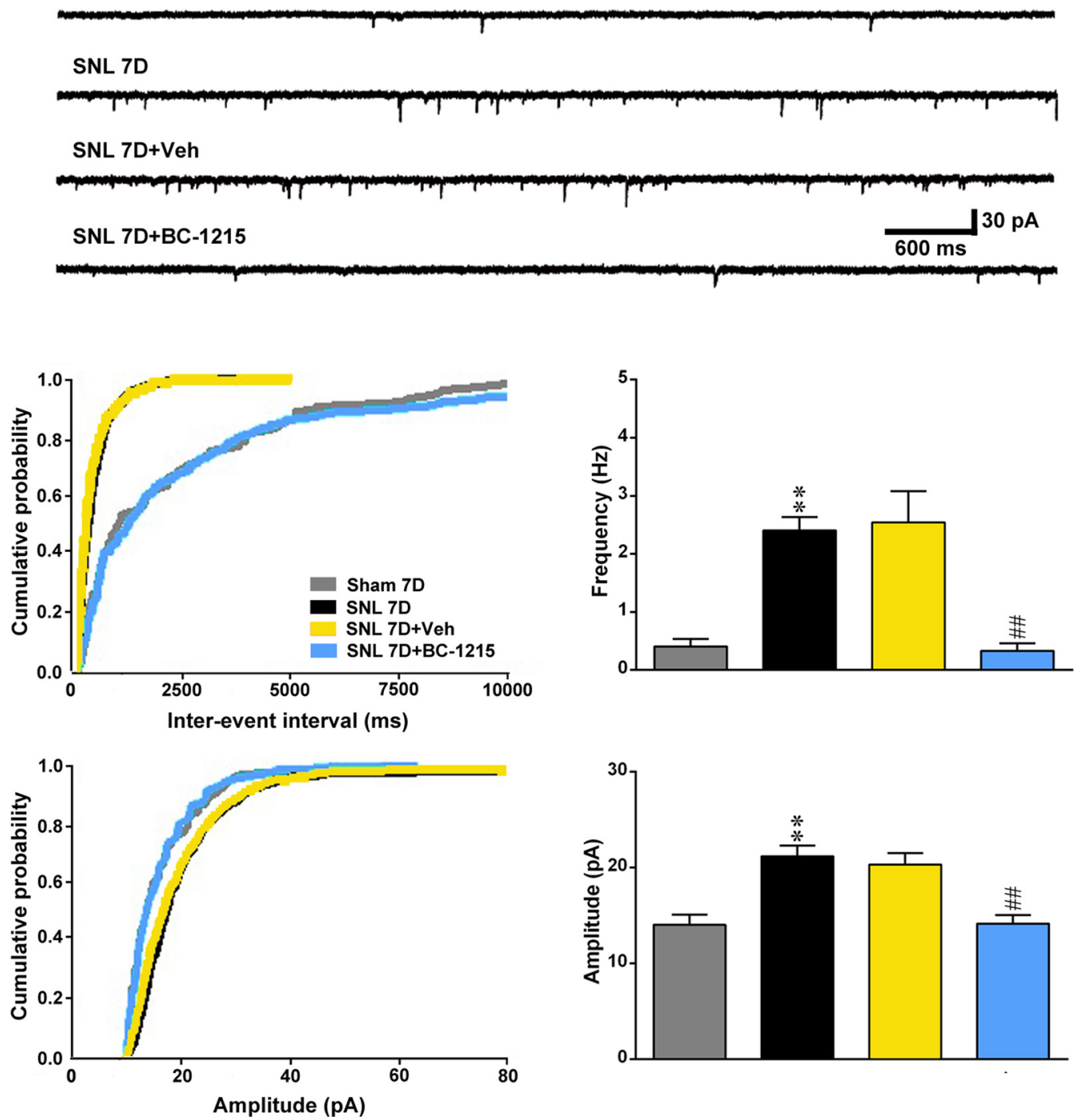

Figure 6. BC-1215 decreases the SNL-enhanced frequency and amplitude of sEPSCs in the ipsilateral dorsal horn. Top, Representative traces of sEPSCS recorded in the ipsilateral dorsal horn neurons of a spinal slice isolated from animals receiving the sham operation (Sham 7D), SNL (SNL 7D), SNL treated with vehicle (SNL 7D + Veh), and BC-1215 (SNL 7D+BC-1215). Lower left, Cumulative probability histograms of the interevent interval (left) and the mean value (right) of the frequency and amplitude of sEPSCs recorded from all groups. Note that the interevent interval of $s$ EPSCs was significantly longer and the amplitude was smaller in the SNL $+B C-1215$ group than in the SNL group $\left(p<0.01\right.$, Kolmogorov-Smirnov test). Average frequency $\left(F_{(3,16)}=14.5\right.$, one-way ANOVA, $p<0.001$, post hoc Tukey's tests; ${ }^{* *} p<0.01$ vs Sham 7D; \#\#p $<0.01$ vs SNL 7D) and amplitude $\left(F_{(3,16)}=12.21\right.$, one-way AN0VA, $p<0.01$, post hoc Tukey's tests; ${ }^{* *} p<0.01$ vs Sham 7D; \#\#p <0.01 vs SNL) of sEPSCs in animals receiving the sham operation, SNL, SNL with vehicle, and BC-1215.

$\mathrm{Ca}_{\mathrm{V}} 2.2$, which underlies fast excitation-secretion coupling (Han et al., 2011; Fernández-Busnadiego et al., 2013). Notably, in the spinal dorsal horn, $\mathrm{Ca}_{\mathrm{V}} 2.2 \mathrm{~s}$ are specially localized in the presynaptic nerve terminals of primary afferents, where they control $\mathrm{Ca}^{2+}$ influx, transmitter release, and the transmission of noxious signals to the CNS (Lopez Soto and Raingo, 2012). Moreover, previous studies have demonstrated that $\omega$-conotoxin, a selective $\mathrm{Ca}_{\mathrm{V}} 2.2$ blocker, dose-dependently reduces neuropathic allodynia in rats (Jayamanne et al., 2013). We therefore hypothesized that RIM1 $\alpha$ and $\mathrm{Ca}_{\mathrm{V}} 2.2$ work cooperatively in the spinal presynaptic sites to cause SNL-induced neuropathic pain. To this end, we first examined RIM1 $\alpha-\mathrm{Ca}_{\mathrm{V}} 2.2$ interactions in the spinal SPM after SNL using immunoprecipitation analysis. When compared with the sham operation, SNL enhanced the abundance of RIM1 $\alpha$-bound RIM1a $(0.66 \pm 0.04, n=6)$ and $\mathrm{Ca}_{\mathrm{V}} 2.2$ coprecipitates $(0.69 \pm 0.05, n=6)$ in the SPM of the ipsilateral dorsal horn at day 7 after the operation (SPM IP: RIM1 $\alpha$; Fig. 7A), indicating that the interactions of RIM1 $\alpha$ and $\mathrm{Ca}_{\mathrm{V}} 2.2$ were enhanced in the spinal SPM after SNL. Next, by intrathecally injecting $\omega$-conotoxin GVIA, an $\mathrm{Ca}_{\mathrm{V}} 2.2$ antagonist, into neuropathic animals, we examined whether antagonism of spinal $\mathrm{Ca}_{\mathrm{V}} 2.2$ reduces SNL-induced allodynia. On day 7 post-SNL, intrathecal injection of $\omega$-conotoxin GVIA ( $\omega$-conotoxin; 10,30 , and $100 \mathrm{pm} ; 10 \mu \mathrm{l})$, but not vehicle (10 $\mu \mathrm{l})$, dose-dependently increased the withdrawal threshold of the ipsilateral hindpaw at $1-6 \mathrm{~h}$ postinjection (Fig. $7 B$; 10 pM: $2.25 \pm 0.49,3.34 \pm 0.61,3.05 \pm 0.45,1.71 \pm$ $0.20,1.22 \pm 0.22$, and $1.08 \pm 0.18 \mathrm{~g} ; 30 \mathrm{pM}: 3.42 \pm 0.57,4.28 \pm 0.80$, $4.20 \pm 0.95,3.71 \pm 0.52,2.25 \pm 0.46$, and $2.34 \pm 0.45 \mathrm{~g} ; 100 \mathrm{pM}$ : $4.28 \pm 0.52,7.14 \pm 0.73,5.71 \pm 0.52,4.57 \pm 0.36,4.28 \pm 0.80$, and $4.00 \pm 0.87 \mathrm{~g}$, respectively; $n=7)$. In contrast, spinal injection of $\omega$-conotoxin GVIA $(100 \mathrm{pM}, 10 \mu \mathrm{l})$ at day 7 after SNL exhibited no effect on the mechanical threshold of the contralateral hindpaw measured $2 \mathrm{~h}$ after injection, a time point $\omega$-conotoxin GVIA displayed maximal analgesic effect (Fig. $7 C$ ). Also, at $2 \mathrm{~h}$ after injection, $\omega$-conotoxin GVIA $(100 \mathrm{pM}, 10 \mu \mathrm{l})$ did not affect the mechanical 


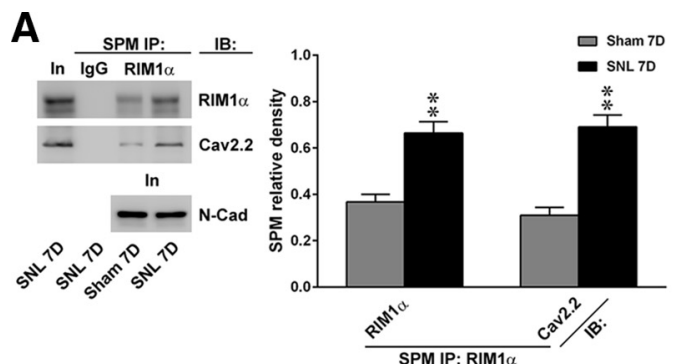

C
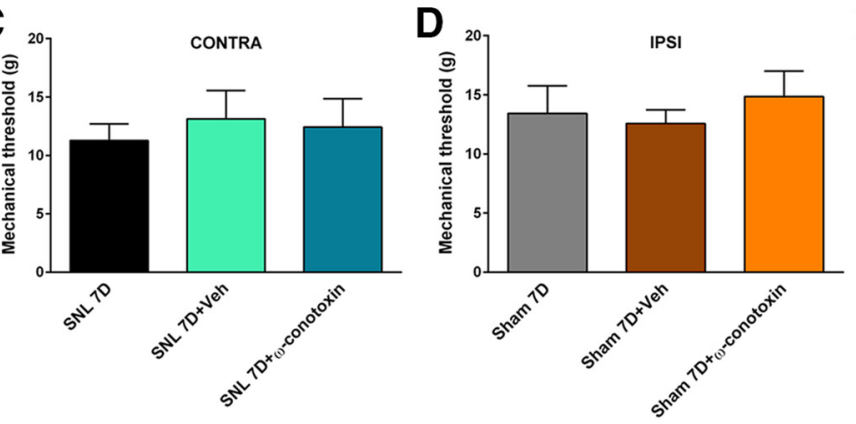

F

Sham 7D
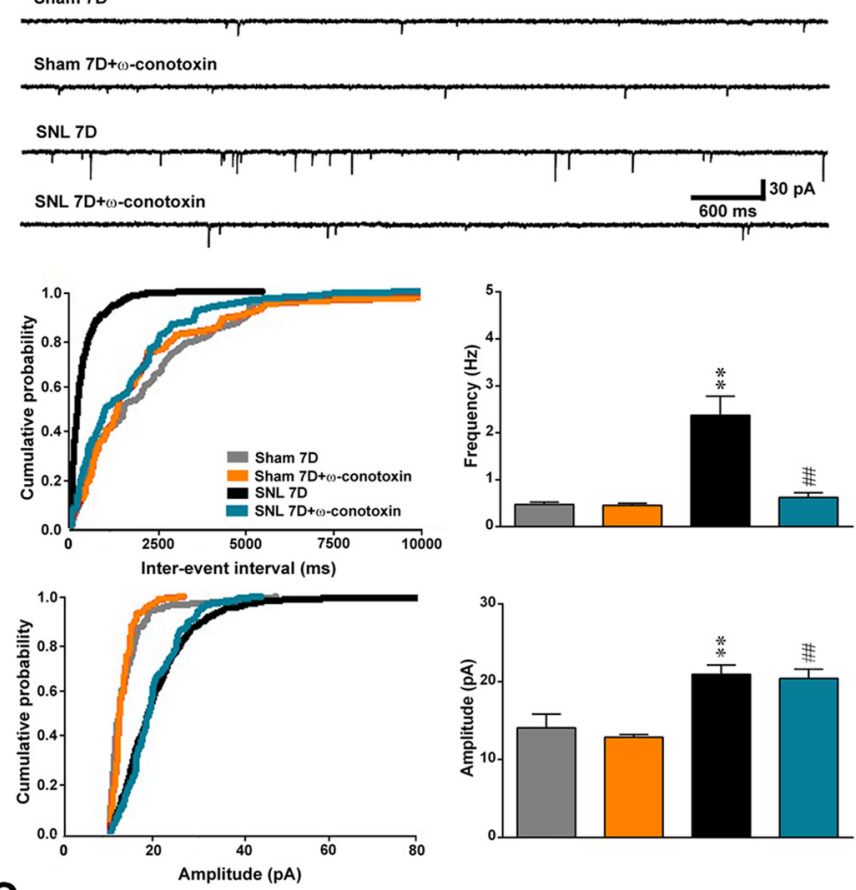

G

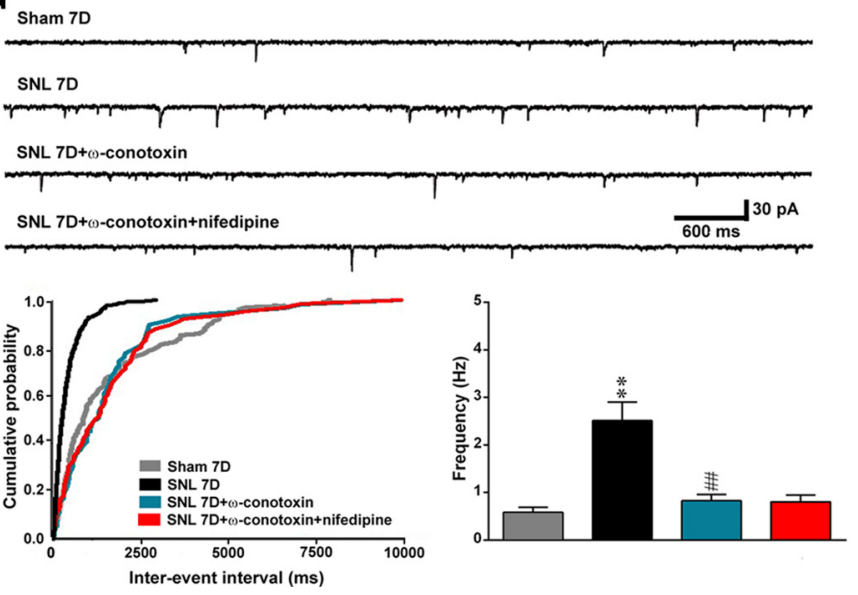

B

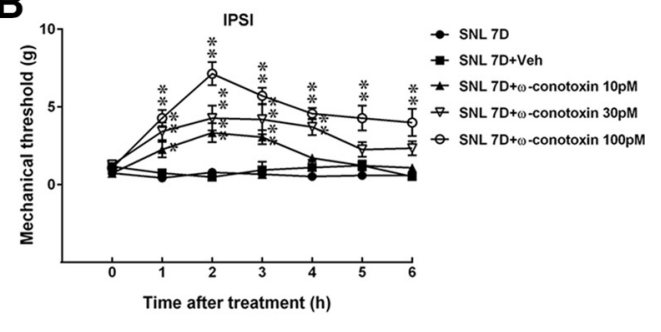

E

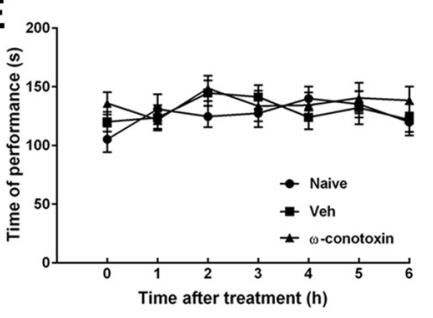


threshold of the ipsilateral hindpaw of sham-operated animals at postoperation day 7 (Fig. 7D). Rotarod analysis showed no significant differences in the motor performance among the naive, vehicletreated, and $\omega$-conotoxin GVIA-treated groups (100 pM, $10 \mu \mathrm{l})$, indicating that the $\omega$-conotoxin GVIA-increased mechanical threshold in SNL animals was not attributed to motor deficits in rats (Fig. 7E). In electrophysiological recordings, $\omega$-conotoxin GVIA $(100 \mathrm{nM})$ also significantly reduced the SNL-induced enhanced frequency, but not amplitude, of sEPSCs (frequency: SNL, $2.37 \pm 0.40$; $n=5$; SNL $+\omega$-conotoxin, $0.62 \pm 0.10 ; n=4$; amplitude: SNL, $21.17 \pm 0.95 ; n=5$; SNL $+\omega$-conotoxin, $20.41 \pm 1.21 ; n=4$; Fig. $7 F)$ in spinal slices. Coapplication of nifedipine $(50 \mu \mathrm{M})$, an L-type postsynaptic calcium channel antagonist, with $\omega$-conotoxin GVIA did not affect the $\omega$-conotoxin GVIA-decreased frequency of sEPSCs in the SNL spinal slices (Fig. 7G). This result suggests that the $\mathrm{Ca}_{\mathrm{V}}$ 2.2-dependent behavioral allodynia that follows neuropathic injury can be attributed to presynaptic mechanisms. Collectively, these data suggest that the RIM $1 \alpha-\mathrm{Ca}_{\mathrm{V}} 2.2$ interaction at the presynaptic membrane of the ipsilateral dorsal horn contributes to the development of neuropathic pain.

\section{$\leftarrow$}

Figure 7. SNL enhances spinal presynaptic RIM1 $\alpha-\mathrm{Ca}_{\mathrm{v}} 2.2$ coupling and increases $C a_{v} 2$ 2-2-dependent mEPSC frequency. $\boldsymbol{A}$, Representative and statistical analysis of immunoprecipitation showing that when compared with sham operation (Sham 7D), SNL (SNL 7D) significantly increased the abundance of RIM1 $\alpha$-bound $\mathrm{Ca}_{\mathrm{v}} 2.2$ in the SPM of ipsilateral dorsal horn samples at day 7 after operation. No detectable immunoreactivity was observed in the control IgG precipitation. IB, Immunoblotting; In, input control; SPM IP, SPM immunoprecipitation. Student's $t$ tests; ${ }^{* *} p<0.01$ vs Sham 7D; $n=6$. B, On postoperative day 7 , a single bolus injection of $\omega$-conotoxin GVIA (SNL 7D $+\omega$-conotoxin; 10,30 , and 100 pm; $10 \mu$ l, i.t.) dose-dependently increased the withdrawal threshold of the ipsilateral hindpaw at hours $1-6$ postinjection (von Frey test). Veh, Vehicle. Two-way ANOVA with repeated measures over time, treatment, $F_{(4,30)}=39.34, p<0.001$; time, $F_{(6,180)}=15.80, p<0.001$; treatment $\times$ time, $F_{(24,180)}=4.374, p<0.001 ;{ }^{*} p<0.05$, ${ }^{* *} p<0.01$ vs SNL 7D; $n=7$. C, When compared with SNL animals, spinal administration with $\omega$-conotoxin GVIA (SNL 7D+ $\omega$-conotoxin, 100 pm; $10 \mu l$ ) did not affect the withdrawal threshold of the contralateral (CONTRA) hindpaw test at $2 \mathrm{~h}$ after injection at post-SNL day 7. One-way ANOVA, $F_{(2,18)}=0.1920, p=0.827$, post hoc Tukey's tests; $n=$ 7. $\boldsymbol{D}$, When compared with sham-operated animals, spinal administration with $\omega$-conotoxin GVIA (100 pm; $10 \mu \mathrm{l}$ ) did not affect the withdrawal threshold of the ipsilateral hindpaw of the sham-operated animals test at $2 \mathrm{~h}$ after injection on post-SNL day 7 . One-way ANOVA, $F_{(2,18)}=0.3498, p=0.709$, post hoc Tukey's tests; $n=7$. $\boldsymbol{E}$, The intrathecal application of neither vehicle solution (10 $\mu$ l) nor $\omega$-conotoxin GVIA (100 pm; $10 \mu l$, i.t.) resulted in motor deficits in rats (rotarod test). Two-way ANOVA with repeated measures over time, treatment, $F_{(2,18)}=1.387, p=0.275$; time, $F_{(6,108)}=1.111, p=$ 0.361 ; treatment $\times$ time, $F_{(12,108)}=0.664, p=0.782 ; n=7 . F$, Top, Representative trace of $s E P S C s$ recorded in the ipsilateral spinal slice isolated from animals receiving the sham operation (Sham 7D), SNL (SNL 7D), as well as sham and SNL treated with $\omega$-conotoxin GVIA (Sham 7D $+\omega$-conotoxin and SNL 7D $+\omega$-conotoxin). Bottom, Cumulative probability histograms of the interevent interval and the amplitude of sEPSCs. Note that the interevent interval of sEPSCs was significantly longer in the SNL $+\omega$-conotoxin group than in the SNL group ( $p<0.01$, Kolmogorov-Smirnov test). Average frequency $\left(F_{(3,14)}=16.81\right.$, one-way ANOVA, $p<0.001$, post hoc Tukey's tests; ${ }^{* *} p<0.01$ vs Sham $7 D ; \# \# p<0.01$ vs SNL) and amplitude $\left(F_{(3,14)}=11.43\right.$, one-way ANOVA, $p<0.01$, post hoc Tukey's tests; ${ }^{* *} p<0.01$ vs Sham 7D) of the sEPSCs in animals receiving the sham operation, SNL, sham treated with $\omega$-conotoxin GVIA (Sham 7D $+\omega$-conotoxin), and SNL treated with $\omega$-conotoxin GVIA (SNL 7D $+\omega$-conotoxin). G, Top, Representative trace of SEPSCs recorded in the ipsilateral spinal slice isolated from animals receiving the sham operation (Sham 7D), SNL (SNL 7D), SNL treated with $\omega$-conotoxin GVIA (SNL 7D+ $\omega$ conotoxin), and SNL treated with $\omega$-conotoxin GVIA and nifedipine (SNL 7D+ $\omega$ conotoxin + nifedipine). Bottom, Cumulative probability histograms of the interevent interval and mean value of the frequency of sEPSCs ( $p<0.01$, Kolmogorov-Smirnov test). Average frequency, $F_{(3,15)}=14.63$, one-way ANOVA, $p<0.001$, post hoc Tukey's tests; ${ }^{* *} p<0.01$ vs Sham 7D; \#\# $<0.01$ vs SNL.

\section{SNL provokes spinal presynaptic}

\section{Fbxo3/Fbxl2/RIM1 $\alpha / C a V 2.2$ signaling}

To further confirm that presynaptic RIM $1 \alpha$ acts as a downstream molecule of the spinal Fbxo3/Fbxl2 cascade to mediate neuropathic pain development by recruiting and coupling with $\mathrm{Ca}_{\mathrm{V}} 2.2$ in the SPM of the dorsal horn, we assayed spinal $\mathrm{Ca}_{\mathrm{V}} 2.2$ expression in the SPM after SNL. At postoperative day 7, Western blotting showed that SNL significantly increased $\mathrm{Ca}_{\mathrm{V}} 2.2$ abundance in the SPM of the ipsilateral dorsal horn (from $0.44 \pm 0.03$ to $0.81 \pm 0.03 ; n=6$; Fig. $8 A$ ), which could be blunted by a single bolus injection of BC-1215 (100 nM, $10 \mu$ l, i.t.; from $0.82 \pm 0.03$ to $0.56 \pm 0.06, n=6$; Fig. $8 B$ ), suggesting that short-term abrogation of spinal presynaptic Fbxo3-mediated Fbxl2 ubiquitination decreased the amount of SNL-upregulated $\mathrm{Ca}_{\mathrm{V}} 2.2$ in the SPM. We next investigated whether spinal Fbxo3 regulates $\mathrm{Ca}_{\mathrm{V}} 2.2$ expression in the SPM via Fbxl2-mediated RIM1 $\alpha$ ubiquitination. Daily administration of RIM $1 \alpha$ mRNA-targeting siRNA ( $5 \mu \mathrm{g}, 10 \mu \mathrm{l}$, i.t.) significantly attenuated SNL-enhanced $\mathrm{Ca}_{\mathrm{V}} 2.2$ expression in the SPM of the ipsilateral dorsal horn at day 7 after the operation (from $0.82 \pm 0.04$ to $0.52 \pm 0.05, n=6$; Fig. $8 C$ ). Single bolus injections of BC-1215 (100 nM, $10 \mu$ l, i.t.) sufficiently decreased the SNL-enhanced abundance of RIM1 $\alpha$ bound RIM $1 \alpha$ (from $0.80 \pm 0.03$ to $0.57 \pm 0.02, n=6$ ) and $\mathrm{Ca}_{\mathrm{V}} 2.2$ (from $0.87 \pm 0.02$ to $0.67 \pm 0.02, \mathrm{n}=6$ ), and of $\mathrm{Ca}_{\mathrm{V}} 2.2-$ bound $\mathrm{Ca}_{\mathrm{V}} 2.2$ (from $0.69 \pm 0.02$ to $0.38 \pm 0.02, n=6$ ) and RIM $1 \alpha$ (from $0.68 \pm 0.05$ to $0.49 \pm 0.04, n=6$ ) in the SPM purified from the ipsilateral dorsal horn samples dissected on day 7 after SNL (Fig. 8D). Conversely, neither intrathecal administration with $\omega$-conotoxin GVIA (100 pM, $10 \mu$ l, i.t.) nor vehicle affected the Fbxo3, Fbxl2, or RIM1 $\alpha$ expression in the SPM (Fig. $8 E)$. Moreover, when compared with BC-1215 injection alone ( $100 \mathrm{nM}, 10 \mu$ l, i.t.; $5.42 \pm 0.57 \mathrm{~g}, n=7$ ), coadministration with $\omega$-conotoxin GVIA ( 100 pM, $10 \mu$ l, i.t.; $6.00 \pm 0.43$ g, $n=7)$ exhibited no additional analgesic effect because there was no statistical difference in withdrawal threshold between these groups (Fig. $8 F$ ). Together, these data support our hypothesis that RIM $1 \alpha$ functions as a downstream molecule of the spinal Fbxo3/ Fbxl2 cascade to mediate neuropathic pain development by recruiting and coupling with $\mathrm{Ca}_{\mathrm{V}} 2.2$ at the presynaptic SPM of the ipsilateral dorsal horn.

\section{Discussion}

In the current study, we report neuropathic injury induces nociceptive hypersensitivity associated with enhanced RIM1 $\alpha$ expression in the pre-SPMs of the dorsal horn. SNL-enhanced Fbxo3 expression increases RIM $1 \alpha$ expression through ubiquitinationdependent Fbxl2 degradation in pre-SPMs. Moreover, the SNLinduced RIM $\alpha$ subsequently interacts with $\mathrm{Ca}_{\mathrm{V}} 2.2$, thereby enhancing the amount of pre-SPM-bound $\mathrm{Ca}_{\mathrm{V}} 2.2$ in the dorsal horn, which underlies the plasticity mediating the development of behavioral allodynia. Our results for the first time report the contribution of spinal $\operatorname{RIM} 1 \alpha$, an active zone protein, to the pathogenesis of neuropathic pain.

RIM1 $\alpha$, the major isoform of mammalian RIM families, was shown to bind with synaptic proteins to mediate vesicle release (Wang et al., 1997, 2000; Kaeser and Südhof, 2005). At synapses, RIM1 $\alpha$ facilitates neurotransmitter release presumably by prompting vesicle priming and fusion (Calakos et al., 2004). In contrast to the deletion of both RIM1 $\alpha$ and $R I M 1 \beta$, which severely impairs mouse survival, the deletion of RIM1 $\alpha$ alone is not lethal and is sufficient to impair synaptic strength and plasticity, i.e., exhibits similar effects as the double deletion of RIM1 $\alpha$ and RIM1 $\beta$ (Kaeser et al., 2008). This finding prompts us to identify 
A
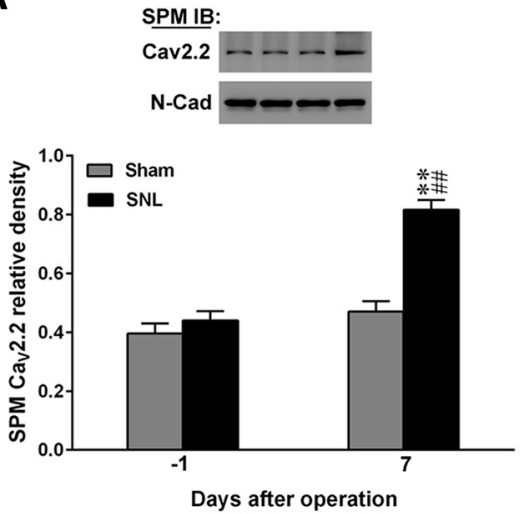

C

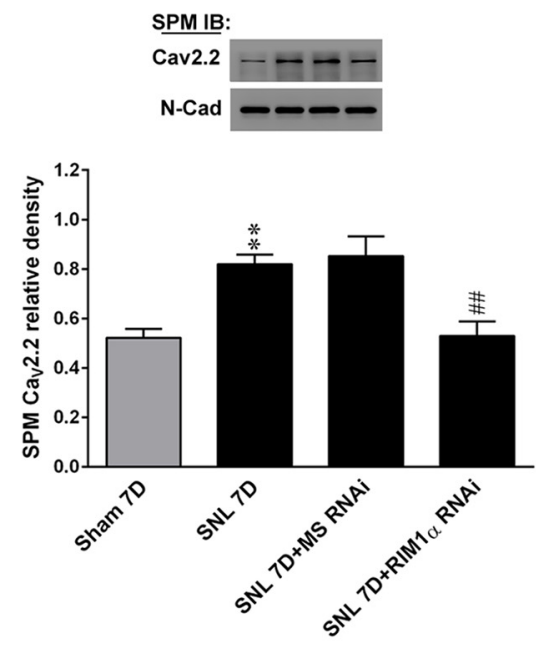

B

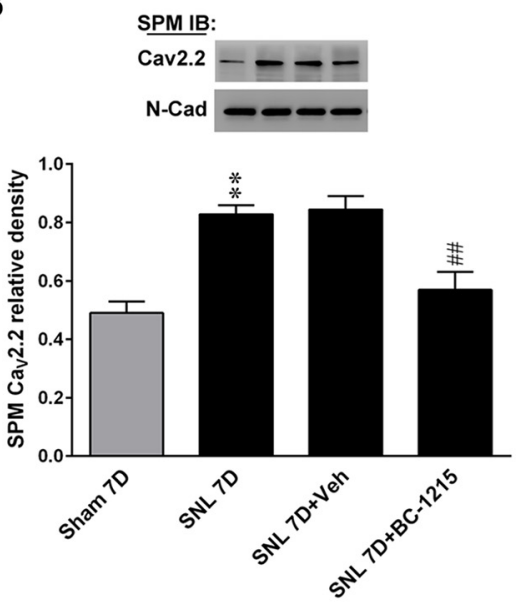

D
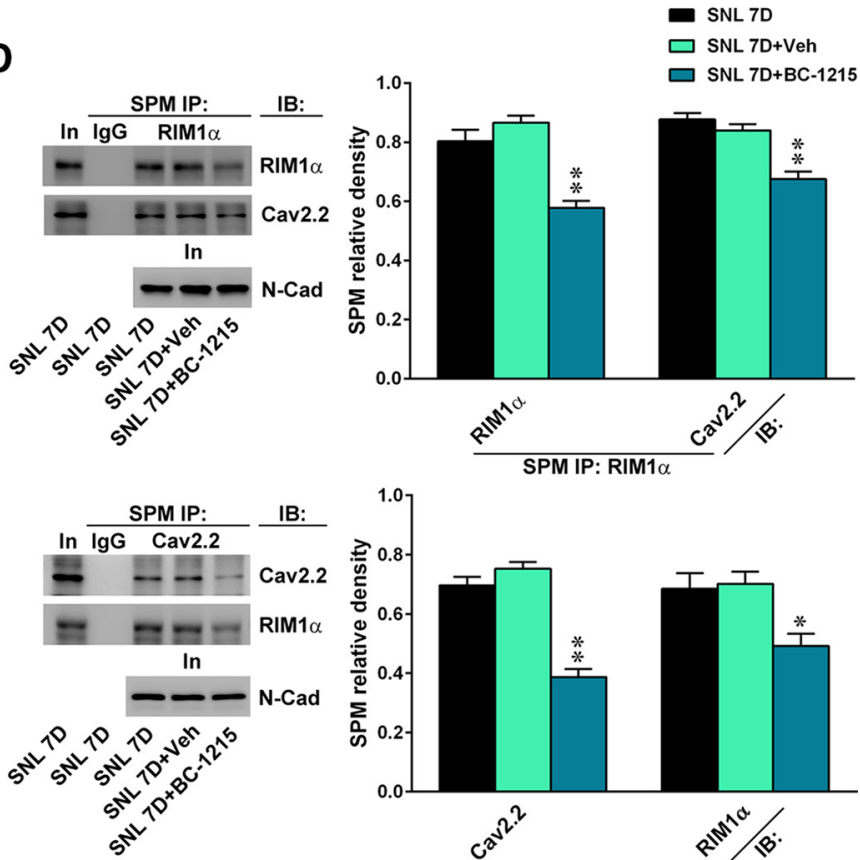

$\mathbf{E}$

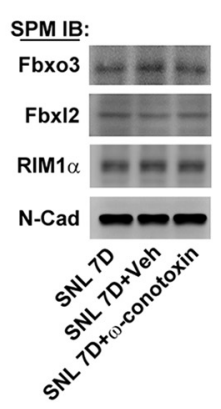

$\square$ SNL 7D

$\square$ SNL 7D+Veh

$\square$ SNL 7D+ $\omega$-conotoxin

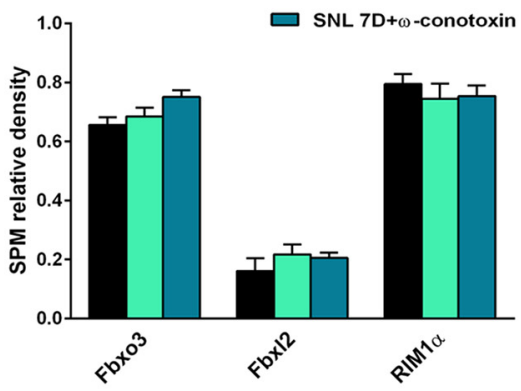

$\mathbf{F}$

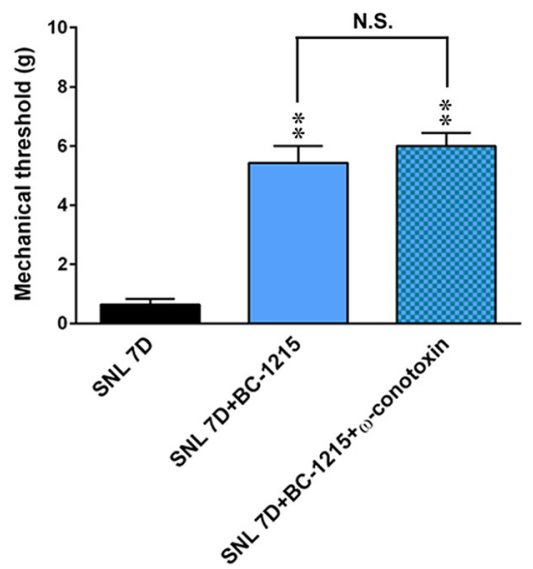

Figure 8. SNL provokes spinal presynaptic Fbx03/FbxI2/RIM1 $\alpha / \mathrm{Ca}_{\mathrm{v}} 2.2$ signaling. A, Representative Western blotting and statistical analysis (normalized to N-cadherin) revealing SNL-increased Cav 2.2 expression in the SPMs of the ipsilateral dorsal horn on day 7 after the operation. SPM IB, SPM immunoblotting. Student's $t$ test; ${ }^{* *} p<0.01$ vs Sham 7D; \#\#p $<0.01$ vs SNL $-1 D ; n=6 . B, A$ single bolus injection of BC-1215 (SNL 7D + BC-1215; $100 \mathrm{~nm}, 10 \mu \mathrm{l}$ ) significantly reversed SNL-enhanced $\mathrm{Ca}_{\mathrm{v}} 2.2$ expression in the SPMs. One-way ANOVA, $F_{(3,20)}=15.53, p<0.001$, post hoc Tukey's tests; ** $p<0.01$ vs Sham 7D; \#\#p $<0.01$ vs SNL 7D; $n=6$. C, The daily administration (days $3-6$ after SNL) of RIM1 $\alpha$ mRNA-targeting siRNA (SNL 7D + RIM1 $\alpha$ RNAi; $5 \mu$, $10 \mu$ l, i.t.) ameliorated SNL-upregulated Cav2.2 expression in the SPMs of the ipsilateral dorsal horn on postoperative day 7. One-way ANOVA, $F_{(3,20)}=10.34$, (Figure legend continues.) 
the role of spinal RIM1 $\alpha$ in the pain pathology. Consistent with evidence linking RIM1 $\alpha$-dependent neurotransmitter release to presynaptic plasticity, our findings further extend the role of RIM1 $\alpha$ in the spinal plasticity underlying neuropathic pain development. Our conclusion is based on the results showing that, besides causing behavioral allodynia, experimental neuropathic injury time-dependently increases the amount of RIM $1 \alpha$ in the total homogenate and SPM of the dorsal horn. Conversely, focal knockdown of spinal RIM1 $\alpha$ expression ameliorates SNLinduced allodynia. Nevertheless, a study demonstrates that RIM1 and RIM2 can replace each other's presynaptic functions at central synapses (Han et al., 2015). Quite different from that study, we observed antisense RNAi dose-dependently decreased spinal RIM1 $\alpha$ but has a minor effect on RIM2 expression. We speculate the RIM1-RIM2 alteration occurs only when the RIM1 function is totally eliminated.

Recently, E3-ubiquitin ligase-mediated protein ubiquitination has attracted researchers' attention for it has a crucial role in regulating the turnover of pain-associated synaptic proteins (Ehlers, 2003; Ossipov et al., 2007). Along with studies linking presynaptic proteins to the pain-associated synaptic plasticity (Yan et al., 2013; Hung et al., 2014), our findings reveal a novel machinery in which ubiquitination-dependent RIM1 $\alpha$ turnover contributes to the development of neuropathic pain. Studies have demonstrated that reagents, such as MG-132, that antagonize protein ubiquitination and thereby the subsequent degradation can attenuate nociceptive responses in arthritic rats (Ahmed et al., 2010). Therefore, it is plausible that antagonizing protein ubiquitination/degradation could relieve pain. Nevertheless, our present data revealed that Fbxo3 modifies spinal RIM1 $\alpha$ expression by mediating Fbxl2 ubiquitination, and that Fbxl2 also inhibits RIM1 $\alpha$ protein via ubiquitination and degradation of RIM $1 \alpha$. It is possible that the application of wide-range ubiquitination inhibitors might produce complicated effects in these ubiquitination pathways. Moreover, systemic reduction of protein ubiquitination, even restricted to the CNS, could result in unopposed effects because specific ubiquitination defects are linked to several neural developmental disorders (Kishino et al., 1997). In contrast, our findings revealed a potential therapeutic effect of BC-1215, an Fbox3-selective antagonist, in pain development, and hence provide a less harmful strategy for the relief of neuropathic pain.

\footnotetext{
(Figure legend continued.) $\quad p<0.001$, post hoc Tukey's tests; ${ }^{* *} p<0.01$ vs Sham 7D; \#\#p $<$ 0.01 vs SNL 7D; $n=6 . \boldsymbol{D}$, Representative and statistical analysis of immunoprecipitation showing that on postoperative day 7, the amount of RIM1 $\alpha$-bound $\mathrm{Ca}_{\mathrm{v}} 2.2$ and $\mathrm{Ca}_{\mathrm{v}} 2.2$-bound $\mathrm{RIM} 1 \alpha$ in the SPMs was reduced by a single bolus injection of $\mathrm{BC}-1215(100 \mathrm{~nm}, 10 \mu \mathrm{l})$. The amount of $\mathrm{N}$-cadherin in the preprecipitated homogenates remained relatively constant at these time points. No detectable immunoreactivity was observed in the control lgG-recognized precipitation. In, Input control. SPM IP, SPM immunoprecipitation. RIM1 $\alpha$-RIM1 $\alpha$, one-way ANOVA, $F_{(2,15)}=25.32, p<0.001$, post hoc Tukey's tests; RIM1 $\alpha$-Ca 2.2 , one-way ANOVA, $F_{(2,15)}=$ $21.99, p<0.001$, posthoc Tukey's tests; $\mathrm{Ca}_{\mathrm{v}} 2.2-\mathrm{Ca}_{\mathrm{v}} 2.2$, one-way ANOVA, $F_{(2,15)}=54.65, p<$ 0.001 , post hoc Tukey's tests; Ca $2.2-R I M 1 \alpha$, one-way ANOVA, $F_{(2,15)}=6.67, p=0.008$, post hoc Tukey's tests; ${ }^{*} p<0.05,{ }^{* *} p<0.01$ vs SNL 7D; $n=6$. $E$, The administration of neither $\omega$-conotoxin GVIA (SNL 7D + $\omega$-conotoxin; 100 pm; $10 \mu$ l, i.t.) nor vehicle (SNL 7D + Veh, 10 $\mu$ ) affected spinal Fbx03, Fbx12, or RIM1 $\alpha$ expression in the SPMs of the ipsilateral dorsal horn on day 7 after SNL. Fbxo3, one-way ANOVA, $F_{(2,15)}=3.41, p=0.06$, post hoc Tukey's tests; Fbxl2, one-way ANOVA, $F_{(2,15)}=0.79, p=0.47$, post hoc Tukey's tests; RIM1 $\alpha$, one-way ANOVA, $F_{(2,15)}=0.41, p=0.67$, post hoc Tukey's tests; $n=6 . F$, When compared with shamoperated animals, spinal BC-1215 injection significantly increased the withdrawal threshold of the ipsilateral hindpaw of SNL animals (SNL 7D + BC-1215, $100 \mathrm{nM} ; 10 \mu$ ), and coadministration with $\omega$-conotoxin GVIA (100 pM; $10 \mu$ l) exhibited no further increase in the withdrawal threshold (SNL $7 \mathrm{D}+\mathrm{BC}-1215+\omega$-conotoxin). ${ }^{* *} p<0.01$ vs SNL 7D; $n=7$.
}

It is worth noting that in this study, a bolus injection of BC1215 had no effect on SNL-induced Fbxo3 expression in the SPM of the dorsal horn, yet it sufficiently ameliorated the associated allodynia. Although the underlying causes remain unclear, we speculated that BC-1215, a reagent originally designed to prevent Skp1-Cullin-Fbxo3-catalysis of Fbxl2 ubiquitination, failed to reverse the SNL-enhanced Fbox3 levels because it blocks Fbxo3 activity but exhibits no effect on Fbxo3 expression (Mallampalli et al., 2013). Similarly, our previous study (Lin et al., 2015b) found a single BC-1215 injection reversed the SNL-associated allodynia but failed to alter the enhanced spinal Fbxo3 expression. Nevertheless, in that study, a bolus of BC-1215 reversed intrathecal TNF $\alpha$ injectioninduced allodynia and spinal Fbxo3 expression. More studies are needed to determine whether BC1215 has a short-lived effect on the pathological enhancement of Fbxo3 expression and whether the long-term administration of BC-1215 could block neuropathic injuryupregulated spinal Fbxo3 expression.

Synaptic protein ubiquitination is implicated in the regulation of both presynaptic and postsynaptic plasticity (Hegde et al., 1997; Ehlers, 2003; Speese et al., 2003; Zhao et al., 2003). In the current study, Fbxo3-selective inhibitor significantly reversed the SNL-enhanced frequency and amplitude of sEPSCs, suggesting the Fbxo3-dependent allodynia originates from the presynaptic and postsynaptic sites of the dorsal horn. While other studies have demonstrated that Fbxo3-dependent ubiquitination mediates pain-associated plasticity by affecting the postsynaptic components (Lin et al., 2015b), the current study reveals a potential involvement of Fbxo3-dependent ubiquitination of presynaptic components in the spinal plasticity underlying neuropathic pain. Nevertheless, a recent study demonstrated that MG-132 decreased the paired-pulse facilitation in the CA3-CA1 synapse of hippocampal slices as well as the mEPSC frequency, but not the amplitude, of the hippocampus pyramidal neurons (Yao et al., 2007). Their results, somewhat different from ours, suggest that ubiquitination mechanisms modify neural transmission/plasticity by acting on presynaptic terminals. We propose several potential causes for this discrepancy. First, in contrast to our study investigating the ubiquitination occurring in dorsal horn neurons, their study recorded neuronal activity in hippocampus slices/neurons. Whether the ubiquitination-dependent modification of neural transmission/plasticity has site-specific effects needs to be clarified. Additionally, MG-132 is a broad-spectrum ubiquitination inhibitor that generally blocks all ubiquitination pathways, making it a reagent quite different from BC-1215, which is an Fbxo3-selective inhibitor that specifically antagonizes Fbxo3-dependent cascades in our study. Yet the potential involvement of Fbxo3-dependent ubiquitination in the postsynaptic machinery cannot be excluded because E3-ubiquitin ligases are shown to interact and thereby modify the expression of postsynaptic density-95, a major scaffolding protein that tethers postsynaptic receptors to the signal proteins/cytoskeleton and hence affects the associated neural plasticity (Colledge et al., 2003). Thus, the detailed mechanisms need to be verified carefully in further studies.

Besides postsynaptic mechanisms (Liu et al., 2000; Yan et al., 2013), another potential mechanism involved in pain-associated spinal plasticity is modification of the glutamatergic neurotransmission at the presynaptic site, because neuropathic injury increases the frequency of glutamate release from the terminal of damaged sensory afferents (Yan et al., 2013). In the current study, though SNL increased both the frequency and amplitudes of sEPSCs, knockdown of spinal RIM1 $\alpha$ expression selectively reversed 
the SNL-increased frequency, indicating a role of RIM1 $\alpha$ in the presynaptic glutamatergic effect. Nevertheless, it is worth noting that application of an Fbxo3 antagonist reversed both the SNLincreased frequency and amplitude of sEPSCs, i.e., Fbxo3 exhibited presynaptic and postsynaptic glutamatergic effects in mediating pain-associated spinal plasticity. This proposal is further supported by the fact that though $\operatorname{RIM} 1 \alpha$ knockdown reduced SNL-enhanced RIM $1 \alpha$ expression and sEPSC frequency to the level comparable to the sham-operated group, it only partially reversed the SNL-associated allodynia. Conversely, in neuropathic rats, knockdown of RIM1 $\alpha$ expression exhibited no significant effect on the BC-1215-exhibited analgesic effect. Additionally, focal trim-down of spinal RIM $1 \alpha$ expression only incompletely reversed the Fbxl2 siRNA-decreased withdrawal threshold. Together with our previous findings showing that Fbxo3/Fbxl2 signaling affected postsynaptic spinal AMPAreceptor expression to underlie the neuropathic pain development (Lin et al., 2015b), our results reveal highly coordinated presynaptic and postsynaptic changes that are additive to increase synaptic strength, and this may be mediated by common upstream signaling subsequent to neuropathic injury. Hence, besides developing pharmacological agents targeting postsynaptic glutamatergic transmission, we identify the presynaptic terminal as a potential site for the medical treatment of neuropathic pain through the use of targeting proteins that regulate glutamate release, such as Fbxo3 and RIM1 $\alpha$.

$\mathrm{Ca}_{\mathrm{V}} 2.2$ is recognized to be localized in the presynaptic terminals and hence contributes to glutamatergic neurotransmission (Bobich et al., 2004). On day 7 after SNL, we observed the experimental neuropathic injury-upregulated spinal $\mathrm{Ca}_{\mathrm{V}} 2.2$ expression and coupling with $\operatorname{RIM} 1 \alpha$, a recognized presynaptic active zone protein, in the SPM. Moreover, spinal injection of $\mathrm{Ca}_{\mathrm{V}} 2.2$ antagonist $\omega$-conotoxin GVIA significantly reduced the SNLenhanced frequency but not amplitude of sEPSCs. This evidence implies a role for presynaptic $\mathrm{Ca}_{\mathrm{V}} 2.2$ in the spinal plasticity underlying the development of neuropathic pain. This speculation is further supported by the fact that coadministration with nifedipine, a postsynaptic calcium channel antagonist, exhibited no additional effect on the decrease in frequency of sEPSCs in dorsal horn neurons of SNL animals caused by the $\omega$-conotoxin GVIA.

We observed that the experimental neuropathic injuryupregulated spinal $\mathrm{Ca}_{\mathrm{V}} 2.2$ expression in the SPM was significantly attenuated by a bolus injection of $\mathrm{BC}-1215$, revealing a prompt Fbxo3 ubiquitination-modified $\mathrm{Ca}_{\mathrm{V}} 2.2$ increase in the SPM of the dorsal horn. Our findings are consistent with a previous study reporting that protein ubiquitination/degradation dynamically regulates $\mathrm{Ca}_{\mathrm{V}} 2.2$ expression at the cell surface (Gandini et al., 2014), and with a study reporting that ubiquitination acutely modifies presynaptic neurotransmitter release in mammalian neurons (Rinetti and Schweizer, 2010). Nevertheless, while BC-1215 significantly increased the withdrawal threshold, coadministration with $\omega$-conotoxin GVIA exhibits no further analgesic effect on the SNL rat. Together with evidence discussed, this result suggests that presynaptic $\mathrm{Ca}_{\mathrm{V}} 2.2$ plays a role as one of the possible downstream molecules of the Fbox 3 in mediating development of neuropathic pain.

\section{References}

Ahmed AS, Li J, Ahmed M, Hua L, Yakovleva T, Ossipov MH, Bakalkin G, Stark A (2010) Attenuation of pain and inflammation in adjuvantinduced arthritis by the proteasome inhibitor MG132. Arthritis Rheum 62:2160-2169. CrossRef Medline

Bobich JA, Zheng Q, Campbell A (2004) Incubation of nerve endings with a physiological concentration of Abeta1-42 activates CaV2.2(N-Type)- voltage operated calcium channels and acutely increases glutamate and noradrenaline release. J Alzheimers Dis 6:243-255. Medline

Butz S, Fernandez-Chacon R, Schmitz F, Jahn R, Südhof TC (1999) The subcellular localizations of atypical synaptotagmins III and VI. Synaptotagmin III is enriched in synapses and synaptic plasma membranes but not in synaptic vesicles. J Biol Chem 274:18290-18296. CrossRef Medline

Calakos N, Schoch S, Südhof TC, Malenka RC (2004) Multiple roles for the active zone protein RIMlalpha in late stages of neurotransmitter release. Neuron 42:889-896. CrossRef Medline

Chen BB, Coon TA, Glasser JR, McVerry BJ, Zhao J, Zhao Y, Zou C, Ellis B, Sciurba FC, Zhang Y, Mallampalli RK (2013) A combinatorial F box protein directed pathway controls TRAF adaptor stability to regulate inflammation. Nat Immunol 14:470-479. CrossRef Medline

Chung JM, Kim HK, Chung K (2004) Segmental spinal nerve ligation model of neuropathic pain. Methods Mol Med 99:35-45. Medline

Colledge M, Snyder EM, Crozier RA, Soderling JA, Jin Y, Langeberg LK, Lu H, Bear MF, Scott JD (2003) Ubiquitination regulates PSD-95 degradation and AMPA receptor surface expression. Neuron 40:595-607. CrossRef Medline

Coppola T, Magnin-Luthi S, Perret-Menoud V, Gattesco S, Schiavo G, Regazzi R (2001) Direct interaction of the Rab3 effector RIM with Ca2+ channels, SNAP-25, and synaptotagmin. J Biol Chem 276:32756-32762. CrossRef Medline

Ehlers MD (2003) Activity level controls postsynaptic composition and signaling via the ubiquitin-proteasome system. Nat Neurosci 6:231-242. CrossRef Medline

Fernández-Busnadiego R, Asano S, Oprisoreanu AM, Sakata E, Doengi M, Kochovski Z, Zürner M, Stein V, Schoch S, Baumeister W, LucićV (2013) Cryo-electron tomography reveals a critical role of RIMlalpha in synaptic vesicle tethering. J Cell Biol 201:725-740. CrossRef Medline

Gandini MA, Sandoval A, González-Ramírez R, Mori Y, de Waard M, Felix R (2011) Functional coupling of Rab3-interacting molecule 1 (RIM1) and L-type Ca2+ channels in insulin release. J Biol Chem 286:15757-15765. CrossRef Medline

Gandini MA, Henríquez DR, Grimaldo L, Sandoval A, Altier C, Zamponi GW, Felix R, González-Billault C (2014) CaV2.2 channel cell surface expression is regulated by the light chain 1 ( $\mathrm{LC1}$ ) of the microtubuleassociated protein B (MAP1B) via UBE2L3-mediated ubiquitination and degradation. Pflugers Arch 466:2113-2126. CrossRef Medline

Geppert M, Goda Y, Stevens CF, Südhof TC (1997) The small GTP-binding protein Rab3A regulates a late step in synaptic vesicle fusion. Nature 387:810-814. CrossRef Medline

Han Y, Kaeser PS, Südhof TC, Schneggenburger R (2011) RIM determines $\mathrm{Ca}(2)+$ channel density and vesicle docking at the presynaptic active zone. Neuron 69:304-316. CrossRef Medline

Han Y, Babai N, Kaeser P, Südhof TC, Schneggenburger R (2015) RIM1 and RIM2 redundantly determine Ca2+ channel density and readily releasable pool size at a large hindbrain synapse. J Neurophysiol 113:255-263. CrossRef Medline

Hegde AN, Inokuchi K, Pei W, Casadio A, Ghirardi M, Chain DG, Martin KC, Kandel ER, Schwartz JH (1997) Ubiquitin C-terminal hydrolase is an immediate-early gene essential for long-term facilitation in Aplysia. Cell 89:115-126. CrossRef Medline

Hibino H, Pironkova R, Onwumere O, Vologodskaia M, Hudspeth AJ, Lesage F (2002) RIM binding proteins (RBPs) couple Rab3-interacting molecules (RIMs) to voltage-gated $\mathrm{Ca}(2+)$ channels. Neuron 34:411-423. CrossRef Medline

Hung KL, Wang SJ, Wang YC, Chiang TR, Wang CC (2014) Upregulation of presynaptic proteins and protein kinases associated with enhanced glutamate release from axonal terminals (synaptosomes) of the medial prefrontal cortex in rats with neuropathic pain. Pain 155:377-387. CrossRef Medline

Jayamanne A, Jeong HJ, Schroeder CI, Lewis RJ, Christie MJ, Vaughan CW (2013) Spinal actions of omega-conotoxins, CVID, MVIIA and related peptides in a rat neuropathic pain model. Br J Pharmacol 170:245-254. CrossRef Medline

Kaeser PS, Südhof TC (2005) RIM function in short- and long-term synaptic plasticity. Biochem Soc Trans 33:1345-1349. CrossRef Medline

Kaeser PS, Kwon HB, Chiu CQ, Deng L, Castillo PE, SüdhofTC (2008) RIM1alpha and RIM1beta are synthesized from distinct promoters of the RIM1 gene to mediate differential but overlapping synaptic functions. J Neurosci 28:13435-13447. CrossRef Medline 
Kishino T, Lalande M, Wagstaff J (1997) UBE3A/E6-AP mutations cause Angelman syndrome. Nat Genet 15:70-73. CrossRef Medline

Kohno T, Ji RR, Ito N, Allchorne AJ, Befort K, Karchewski LA, Woolf CJ (2005) Peripheral axonal injury results in reduced mu opioid receptor pre- and post-synaptic action in the spinal cord. Pain 117:77-87. CrossRef Medline

Lin TB, Lai CY, Hsieh MC, Jiang JL, Cheng JK, Chau YP, Ruan T, Chen GD, Peng HY (2015a) Neuropathic allodynia involves spinal neurexin1beta-dependent neuroligin-1/postsynaptic density-95/NR2B cascade in rats. Anesthesiology 123:909-926. CrossRef Medline

Lin TB, Hsieh MC, Lai CY, Cheng JK, Chau YP, Ruan T, Chen GD, Peng HY (2015b) Fbxo3-dependent Fbxl2 ubiquitination mediates neuropathic allodynia through the TRAF2/TNIK/GluR1 cascade. J Neurosci 35: 16545-16560. CrossRef Medline

Lin TB, Hsieh MC, Lai CY, Cheng JK, Wang HH, Chau YP, Chen GD, Peng HY (2016) Melatonin relieves neuropathic allodynia through spinal MT2-enhanced PP2Ac and downstream HDAC4 shuttling-dependent epigenetic modification of hmgb1 transcription. J Pineal Res 60:263-276. CrossRef Medline

Liu X, Eschenfelder S, Blenk KH, Jänig W, Häbler H (2000) Spontaneous activity of axotomized afferent neurons after L5 spinal nerve injury in rats. Pain 84:309-318. CrossRef Medline

Lopez Soto EJ, Raingo J (2012) A118G mu opioid receptor polymorphism increases inhibitory effects on CaV2.2 channels. Neurosci Lett 523:190194. CrossRef Medline

Mallampalli RK, Coon TA, Glasser JR, Wang C, Dunn SR, Weathington NM, Zhao J, Zou C, Zhao Y, Chen BB (2013) Targeting F box protein Fbxo3 to control cytokine-driven inflammation. J Immunol 191:5247-5255. CrossRef Medline

Michel K, Müller JA, OprişoreanuAM, Schoch S (2015) The presynaptic active zone: a dynamic scaffold that regulates synaptic efficacy. Exp Cell Res 335:157-164. CrossRef Medline

Ossipov MH, Bazov I, Gardell LR, Kowal J, Yakovleva T, Usynin I, Ekström TJ, Porreca F, Bakalkin G (2007) Control of chronic pain by the ubiquitin proteasome system in the spinal cord. J Neurosci 27:8226-8237. CrossRef Medline

Rinetti GV, Schweizer FE (2010) Ubiquitination acutely regulates presynaptic neurotransmitter release in mammalian neurons. J Neurosci 30:31573166. CrossRef Medline

Saegusa H, Tanabe T (2014) N-type voltage-dependent Ca2+ channel in non-excitable microglial cells in mice is involved in the pathophysiology of neuropathic pain. Biochem Biophys Res Commun 450:142-147. CrossRef Medline

Schäfers M, Svensson CI, Sommer C, Sorkin LS (2003) Tumor necrosis factor-alpha induces mechanical allodynia after spinal nerve ligation by activation of p38 MAPK in primary sensory neurons. J Neurosci 23:25172521. Medline

Schoch S, Castillo PE, Jo T, Mukherjee K, Geppert M, Wang Y, Schmitz F, Malenka RC, Südhof TC (2002) RIM1alpha forms a protein scaffold for regulating neurotransmitter release at the active zone. Nature 415:321326. CrossRef Medline

Schoch S, Mittelstaedt T, Kaeser PS, Padgett D, Feldmann N, Chevaleyre V, Castillo PE, Hammer RE, Han W, Schmitz F, Lin W, Südhof TC (2006) Redundant functions of RIM1alpha and RIM2alpha in $\mathrm{Ca}(2+)$-triggered neurotransmitter release. EMBO J 25:5852-5863. CrossRef Medline

Spangler SA, Schmitz SK, Kevenaar JT, de Graaff E, de Wit H, Demmers J, Toonen RF, Hoogenraad CC (2013) Liprin-alpha2 promotes the presynaptic recruitment and turnover of RIM1/CASK to facilitate synaptic transmission. J Cell Biol 201:915-928. CrossRef Medline

Speese SD, Trotta N, Rodesch CK, Aravamudan B, Broadie K (2003) The ubiquitin proteasome system acutely regulates presynaptic protein turnover and synaptic efficacy. Curr Biol 13:899-910. CrossRef Medline

Varshavsky A (2005) Regulated protein degradation. Trends Biochem Sci 30:283-286. CrossRef Medline

Wang X, Hu B, Zimmermann B, Kilimann MW (2001) Riml and rabphilin-3 bind Rab3-GTP by composite determinants partially related through N-terminal alpha-helix motifs. J Biol Chem 276:32480-32488. CrossRef Medline

Wang Y, Okamoto M, Schmitz F, Hofmann K, Südhof TC (1997) Rim is a putative Rab3 effector in regulating synaptic-vesicle fusion. Nature 388: 593-598. CrossRef Medline

Wang Y, Sugita S, Sudhof TC (2000) The RIM/NIM family of neuronal C2 domain proteins. Interactions with Rab3 and a new class of Src homology 3 domain proteins. J Biol Chem 275:20033-20044. CrossRef Medline

Yan X, Jiang E, Gao M, Weng HR (2013) Endogenous activation of presynaptic NMDA receptors enhances glutamate release from the primary afferents in the spinal dorsal horn in a rat model of neuropathic pain. J Physiol 591:2001-2019. CrossRef Medline

Yao I, Takagi H, Ageta H, Kahyo T, Sato S, Hatanaka K, Fukuda Y, Chiba T, Morone N, Yuasa S, Inokuchi K, Ohtsuka T, Macgregor GR, Tanaka K, Setou M (2007) SCRAPPER-dependent ubiquitination of active zone protein RIM1 regulates synaptic vesicle release. Cell 130:943-957. CrossRef Medline

Yao I, Takao K, Miyakawa T, Ito S, Setou M (2011) Synaptic E3 ligase SCRAPPER in contextual fear conditioning: extensive behavioral phenotyping of Scrapper heterozygote and overexpressing mutant mice. PLoS One 6:e17317. CrossRef Medline

Yi JJ, Ehlers MD (2005) Ubiquitin and protein turnover in synapse function. Neuron 47:629-632. CrossRef Medline

Zhao Y, Hegde AN, Martin KC (2003) The ubiquitin proteasome system functions as an inhibitory constraint on synaptic strengthening. Curr Biol 13:887-898. CrossRef Medline

Zhou HY, Chen SR, Chen H, Pan HL (2010) Opioid-induced long-term potentiation in the spinal cord is a presynaptic event. J Neurosci 30:4460 4466. CrossRef Medline

Zimmermann M (1983) Ethical guidelines for investigations of experimental pain in conscious animals. Pain 16:109-110. CrossRef Medline 